\title{
CONVERGENCE OF MONOTONE SCHEMES FOR CONSERVATION LAWS WITH ZERO-FLUX BOUNDARY CONDITIONS
}

\author{
K. H. KARLSEN ${ }^{\mathrm{A}}$ AND J. D. TOWERS ${ }^{\mathrm{B}}$
}

\begin{abstract}
We consider a scalar conservation law with zero-flux boundary conditions imposed on the boundary of a rectangular multidimensional domain. We study monotone schemes applied to this problem. For the Godunov version of the scheme, we simply set the boundary flux equal to zero. For other monotone schemes, we additionally apply a simple modification to the numerical flux. We show that the approximate solutions produced by these schemes converge to the unique entropy solution, in the sense of [7, of the conservation law. Our convergence result relies on a BV bound on the approximate numerical solution. In addition, we show that a certain functional that is closely related to the total variation is nonincreasing from one time level to the next. We extend our scheme to handle degenerate convection-diffusion equations, and for the one-dimensional case we prove convergence to the unique entropy solution.
\end{abstract}

\section{INTRODUCTION}

We are interested in an initial-boundary value problem of the form

$$
\left\{\begin{array}{l}
u_{t}+\nabla \cdot f(u):=u_{t}+\sum_{i=1}^{d} f_{i}(u)_{x_{i}}=0, \quad\left(x_{1}, \ldots, x_{d}\right) \in \Omega, \quad t \in(0, T), \\
f(u(x, t)) \cdot \nu=0 \text { a.e. on } \partial \Omega \times(0, T), \\
u(x, 0)=u_{0}(x), \quad x \in \Omega .
\end{array}\right.
$$

Here $\Omega=\Pi_{i=1}^{d}\left(0, a_{i}\right)$ is an open rectangular region in $\mathbb{R}^{d}$, and $\nu$ is the a.e. defined outward unit normal vector to the spatial region $\Omega$. We assume the flux functions $u \mapsto f_{i}(u)$ are Lipschitzcontinuous and satisfy $f_{i}(0)=f_{i}(1)=0, f_{i}(u) \geq 0$ for $u \in[0,1]$. We assume that the initial function $u_{0}$ satisfies

$$
u_{0} \in L^{1}(\Omega) \cap \mathrm{BV}(\Omega) ; \quad u_{0}(x) \in[0,1] \quad \forall x \in \Omega .
$$

The well-posedness of the Cauchy problem corresponding to 1.1 was established by Kružkov [19]. The Dirichlet problem, where the conserved quantity $u$ is specified on the spatial boundary, has also been well understood for a long time [4. On the other hand, the study of problem (1.1), which specifies zero flux through the spatial boundary, did not begin until more recently.

Problems like (1.1) occur in several applications, including porous media flow, sedimentation processes, and road traffic. For example, batch or continuous sedimentation processes are utilized in many industrial applications in which a solid-fluid suspension is separated into its solid and fluid components under the influence of gravity. Relevant models often give rise to hyperbolic (or degenerate parabolic) equations with the zero flux (homogeneous Neumann) boundary condition. For examples of such applications in the one-dimensional setting, see, e.g., [5, 8, 10].

Karlsen, Lie, and Risebro 18 proposed a front tracking algorithm for producing approximate solutions to (1.1). For the one-dimensional case, they proved that the front tracking approximations converge to a unique weak solution. Their convergence proof relied on a total variation bound. They also proposed a front tracking algorithm for the multidimensional version of the

\footnotetext{
Date: May 29, 2016.

Key words and phrases. degenerate parabolic equation, scalar conservation law, zero-flux boundary condition, monotone scheme, convergence.

A Department of Mathematics, University of Oslo, P.O. Box 1053, Blindern, N-0316 Oslo, Norway. E-Mail: kennethk@math.uio.no.

B MiraCosta College, 3333 Manchester Avenue, Cardiff-by-the-Sea, CA 92007-1516, USA.

E-mail:john.towers@cox.net.
} 
problem, using dimensional splitting. They did not prove convergence of their multidimensional scheme, the main obstacle being the lack of a total variation bound. The authors of 7 studied the multidimensional version of the problem, allowing for a fairly general boundary (specifically, a regular deformable Lipschitz boundary). They also proposed a definition of $L^{\infty}$ entropy solution, which we have adopted below for the special case of a rectangular boundary. Bürger, Frid and Karlsen considered a sequence of regularized parabolic problems, and proved convergence to a unique entropy solution, using the compactness result of 20. In both 18 and 7], the authors mentioned the seeming lack of a BV bound in the multidimensional case. A significant extension of the results in [7] to general boundary value problems can be found in Andreianov and Sbihi [3]. These authors consider conservation laws with a general dissipative boundary condition, which includes as particular cases the Dirichlet, Neumann (flux), Robin, and obstacle boundary conditions. Well-posedness results for degenerate parabolic problems have been provided by Andreianov and Gazibo [1, 2].

For the Cauchy problem, the theory of monotone schemes has been well established for a long time [11, 15, 21]. For the Cauchy problem again, but with a degenerate diffusion term included, the theory of monotone schemes was addressed more recently [13].

To our knowledge, there are no previous published results on the subject of convergence of finite difference schemes for the zero-flux boundary problem (1.1), even for the important onedimensional case. However, there is a recent convergence result for an implicit finite volume method for degenerate parabolic equations (with zero flux condition), due to Andreianov and Gazibo 2]. The convergence proof in [2] is rather involved, relying on sophisticated energy (weak BV) estimates and nonlinear weak convergence techniques. Our proof, on the other hand, is short and elementary, relying on BV estimates obtained through a slight modification of the total variation functional (the only intricate trick involved).

Compared to references [18] and [7], we have added the hypothesis $f_{i}(u) \geq 0$, which is crucial to our total variation bound. This is an entirely natural assumption for applications such as sedimentation in closed vessels, traffic flow, and certain two-phase porous media flow problems. We study monotone schemes as applied to (1.1). Away from the boundary, the schemes are standard as in 11, 15, 21. We handle the boundary condition in the obvious way, setting the numerical flux that is normal to the boundary equal to zero. For schemes other than the Godunov version, we additionally modify the numerical flux slightly, in order keep the flux nonnegative. For the Godunov version, this modification is not required. In any case, the result is a very easily implemented class of numerical schemes.

We prove that the schemes mentioned above produce approximate solutions that converge to the unique entropy solution in the sense of 7 . As part of the convergence proof, we establish a bound on the total variation of the approximate solutions. In fact we prove that a certain functional, denoted $\mathrm{TV}^{*}$, which is closely related to the total variation, is nonincreasing from one time level to the next. Although the scheme is formally first order accurate (as are all monotone schemes), it is potentially a starting point for higher order schemes. In particular, a design goal would be to achieve second (or higher) order formal accuracy while maintaining the property that $\mathrm{TV}^{*}$ is nonincreasing. We extend our scheme to handle the more general case of a degenerate convectiondiffusion equation. We obtain some partial results in that direction, namely that the total variation bound still holds for the extended scheme, and that the extended scheme satisfies a discrete entropy inequality consistent with the ones found in [1] and 14. For the one-dimensional version of the extended scheme, we prove convergence to the unique entropy solution. The analogous result for the multidimensional problem remains open, the main difficulty being the lack of an existence result for the trace of the total flux along the spatial boundary.

The remaining part of this paper is organized as follows: In Section 2 we recall the notion of entropy solution. The difference scheme is defined in Section 3 , and analyzed in Section 4. A few one- and two-dimensional numerical examples are presented in Section 5 . In Section 6 we briefly discuss how the scheme can be modified to handle degenerate convection-diffusion equations. 


\section{Notion OF SOLUTION}

In the sequel, we mostly restrict the presentation to $d=2$, and use the notation $x=x_{1}, y=x_{2}$, $f_{1}=f, f_{2}=g$. The corresponding definitions and results will be clear from this case. Let

$$
Q_{T}:=\Omega \times(0, T), \quad \underline{Q}_{T}:=\Omega \times[0, T), \quad \Pi_{T}:=\mathbb{R}^{d} \times(0, T), \quad \underline{\Pi}_{T}:=\mathbb{R}^{d} \times[0, T),
$$

and denote by $C_{0}^{\infty}\left(Q_{T}\right)$ the set of all infinitely smooth functions on $Q_{T}$ with compact support. The space of test functions $C_{0}^{\infty}\left(\underline{\Pi}_{T}\right)$ is defined analogously.

We will use the following definition of entropy solution, taken from [7] and here adapted to the case of a two-dimensional rectangular domain:

Definition 2.1. A function $u \in L^{\infty}\left(Q_{T}\right)$ is called an entropy solution of the initial-boundary value problem (1.1) if the following conditions are satisfied:

(1) For all $\kappa \in \mathbb{R}$ and all $\phi \in C_{0}^{\infty}\left(Q_{T}\right), \phi \geq 0$, the following entropy inequality holds:

$$
\int_{0}^{T} \int_{\Omega}\left\{|u-\kappa| \phi_{t}+\operatorname{sign}(u-\kappa)(f(u)-f(\kappa)) \phi_{x}+\operatorname{sign}(u-\kappa)(g(u)-g(\kappa)) \phi_{y}\right\} d x d y d t \geq 0 .
$$

(2) The initial condition is satisfied as a limit in the following $L^{1}$ sense:

$$
\underset{t \rightarrow 0^{+}}{\operatorname{ess} \lim _{\Omega}} \int_{\Omega}\left|u(x, y, t)-u_{0}(x, y)\right| d x d y=0 .
$$

(3) The boundary condition is satisfied in the following pointwise sense:

$$
\left(f\left(u^{\tau}\right), g\left(u^{\tau}\right)\right) \cdot \nu=0 \text { a.e. on } \partial \Omega \times(0, T),
$$

where $u^{\tau}$ is the strong trace of $u$.

Remark 2.1. Taking $\kappa=0, \kappa=1$ in the entropy inequality (2.1), the result is

$$
\int_{0}^{T} \int_{\Omega}\left\{u \phi_{t}+f(u) \phi_{x}+g(u) \phi_{y}\right\} d x d y d t=0
$$

meaning that $u$ is a weak solution of the conservation law $u_{t}+f(u)_{x}+g(u)_{y}=0$ in $Q_{T}$.

In view of Definition 2.1, we will need to know that the strong boundary trace $u^{\tau}$ exist. For this purpose, we could adopt (the two-dimensional version of) the genuinely nonlinearity condition of [20]. In the present paper, however, we will manage to construct solutions $u$ with bounded total variation, and with $u \in \operatorname{BV}\left(Q_{T}\right)$ the strong trace $u^{\tau}$ is known to exist [12.

The following is an equivalent definition of entropy solutions [7].

Definition 2.2. A function $u \in L^{\infty}\left(Q_{T}\right)$ is called an entropy solution of the initial-boundary value problem [1.1] if the following entropy inequality holds $\forall \kappa \in \mathbb{R}, \forall \phi \in C_{c}^{\infty}\left(\underline{\Pi}_{T}\right), \phi \geq 0$ :

$$
\begin{gathered}
\int_{0}^{T} \int_{\Omega}\left\{|u-\kappa| \phi_{t}+\operatorname{sign}(u-\kappa)(f(u)-f(\kappa)) \phi_{x}+\operatorname{sign}(u-\kappa)(g(u)-g(\kappa)) \phi_{y}\right\} d x d y d t \\
\quad+\int_{\Omega}\left|u_{0}-\kappa\right| \phi(x, y, 0) d x d y+\int_{0}^{T} \int_{\partial \Omega} \operatorname{sign}\left(u^{\tau}-\kappa\right)(f(\kappa), g(\kappa)) \cdot \nu \phi d \mathcal{H}^{N-1} d t \geq 0,
\end{gathered}
$$

where $u^{\tau}$ is the trace of $u$.

Entropy solutions are unique. More precisely,

Theorem 2.1 ([7, Theorem 3.]). Suppose that $u$ and $v$ are entropy solutions of (1.1) with initial conditions $\left.u\right|_{t=0}=u_{0}(x),\left.v\right|_{t=0}=v_{0}(x)$. Assume that $u_{0}, v_{0} \in L^{\infty}(\Omega)$. Then,

$$
\int_{\Omega}|u(x, t)-v(x, t)| d x \leq \int_{\Omega}\left|u_{0}(x)-v_{0}(x)\right| d x .
$$

In particular, there is at most one entropy solution to the zero flux problem (1.1. 


\section{Difference sCHEMES}

We begin by discretizing $\Omega$. For positive integers $J$ and $K$, define the spatial mesh sizes $\Delta x=a_{1} / J$ and $\Delta y=a_{2} / K$. Let $\mathbb{Z}_{J}=\{1, \ldots, J\}, \mathbb{Z}_{K}=\{1, \ldots, K\}$. Define the grid points

$$
x_{j}=(j-1 / 2) \Delta x, \quad y_{k}=(k-1 / 2) \Delta y, \quad j \in \mathbb{Z}_{J}, \quad k \in \mathbb{Z}_{K} .
$$

We will sometimes use the notation

$$
x_{j \pm \frac{1}{2}}=x_{j} \pm \Delta x / 2, \quad y_{k \pm \frac{1}{2}}=y_{k} \pm \Delta y / 2 .
$$

With this setup, the spatial boundaries coincide with

$$
0=x_{\frac{1}{2}}, \quad a_{1}=x_{J+\frac{1}{2}}, \quad 0=y_{\frac{1}{2}}, \quad a_{2}=y_{K+\frac{1}{2}} .
$$

We define intervals $I_{j}^{x}=\left[x_{j-\frac{1}{2}}, x_{j+\frac{1}{2}}\right), I_{k}^{y}=\left[y_{k-\frac{1}{2}}, y_{k+\frac{1}{2}}\right)$. Similarly, we select a time increment $\Delta t>0$ and discretize the time interval $[0, T]: t_{n}=n \Delta t$ for $n=0, \ldots, N$, where $N=\lfloor T / \Delta t\rfloor$, resulting in the time strips

$$
I^{n}=\left[t_{n}, t_{n+1}\right)
$$

Let $\chi_{j}(x), \chi_{k}(y), \chi^{n}(t)$ be the characteristic functions for the intervals $I_{j}^{x}, I_{k}^{y}, I^{n}$ respectively. Define $\chi_{j, k}^{n}(x, y, t)=\chi_{j}(x) \chi_{k}(y) \chi^{n}(t)$ to be the characteristic function for the rectangle

$$
R_{j, k}^{n}=I_{j}^{x} \times I_{k}^{y} \times I^{n} .
$$

We will use $U_{j, k}^{n}$ to denote the finite difference approximation of $u\left(x_{j}, y_{k}, t^{n}\right)$. We discretize the initial data $u_{0}(x, y)$ via

$$
U_{j, k}^{0}=\frac{1}{\Delta x \Delta y} \int_{I_{j}^{x}} \int_{I_{k}^{y}} u_{0}(x, y) d x d y .
$$

The difference solution $\left\{U_{j, k}^{n}\right\}$ is extended to all of $Q_{T}$ by defining

$$
u^{\Delta}(x, t)=\sum_{n=0}^{N} \sum_{k=1}^{K} \sum_{j=1}^{J} \chi_{j, k}^{n}(x, y, t) U_{j, k}^{n}, \quad(x, y, t) \in Q_{T},
$$

where $\Delta=(\Delta x, \Delta y, \Delta t)$.

Let $\bar{f}(v, u)$ and $\bar{g}(v, u)$ denote two-point numerical fluxes that are consistent with $f(u)$ and $g(u)$. With our notation, the first (second) argument denotes the right (left) state. Recall that a two point flux $\bar{q}(v, u)$ is consistent with $q(u)$ if $\bar{q}(u, u)=q(u)$. The numerical flux $\bar{q}(v, u)$ is monotone if it is nonincreasing with respect to its first argument, nondecreasing with respect to its second argument. We will assume that the numerical fluxes $\bar{f}(v, u)$ and $\bar{g}(v, u)$ are monotone, and also Lipschitz continuous with respect to both variables. Some of the most commonly encountered two-point monotone fluxes are the Godunov flux:

the Lax-Friedrichs flux:

$$
\bar{q}(v, u)= \begin{cases}\min _{w \in[u, v]} q(w), & u \leq v, \\ \max _{w \in[v, u]} q(w), & u>v\end{cases}
$$

$$
\bar{q}(v, u)=\frac{1}{2}(q(u)+q(v))-\frac{\alpha}{2 \lambda}(v-u), \quad \alpha \in(0,1]
$$

and the Engquist-Osher flux:

$$
\bar{q}(v, u)=\frac{1}{2}(q(u)+q(v))-\frac{1}{2} \int_{u}^{v}\left|q^{\prime}(w)\right| d w .
$$

If the flux has the form $q(u)=u w(u)$, where $u \mapsto w(u)$ is nonnegative and nonincreasing on $[0,1]$, the Hilleges-Weidlich flux [16, 9] is also a possibility:

$$
\bar{q}(v, u)=u w(v) .
$$

We will employ the following modified flux

$$
\hat{q}(v, u)=\max (0, q(v, u)) .
$$


It is readily verified that $\hat{q}$ is monotone, Lipschitz continuous (with Lipschitz constants not exceeding those of $\bar{q}$ ), and consistent with $q$. Note that for the special cases where $\bar{q}$ is the Godunov flux or the Hilleges-Weidlich flux, $\hat{q}=\bar{q}$.

To simplify the presentation, we use $\Delta_{+}^{x}$ and $\Delta_{-}^{x}$ to designate the difference operators in the $x$ direction, e.g.,

$$
\Delta_{+}^{x} U_{j, k}^{n}=U_{j+1, k}^{n}-U_{j, k}^{n}=\Delta_{-}^{x} U_{j+1, k}^{n},
$$

and $\Delta_{+}^{y}$ and $\Delta_{-}^{y}$ are defined similarly.

With $\lambda^{x}(\Delta)=\Delta t / \Delta x, \lambda^{y}(\Delta)=\Delta t / \Delta y$, the algorithm takes the following (conservation) form

$$
\begin{gathered}
\left\{\begin{array}{c}
V_{j, k}^{n+1}=U_{j, k}^{n}-2 \lambda^{x} \Delta_{-}^{x} \hat{f}\left(U_{j+1, k}^{n}, U_{j, k}^{n}\right), \quad 2 \leq j \leq J-1,1 \leq k \leq K, \\
V_{1, k}^{n+1}=U_{1, k}^{n}-2 \lambda^{x} \hat{f}\left(U_{2, k}^{n}, U_{1, k}^{n}\right), \quad 1 \leq k \leq K, \\
V_{J, k}^{n+1}=U_{J, k}^{n}+2 \lambda^{x} \hat{f}\left(U_{J, k}^{n}, U_{J-1, k}^{n}\right), \quad 1 \leq k \leq K .
\end{array}\right. \\
\left\{\begin{array}{c}
W_{j, k}^{n+1}=U_{j, k}^{n}-2 \lambda^{y} \Delta_{-}^{y} \hat{g}\left(U_{j, k+1}^{n}, U_{j, k}^{n}\right), \quad 2 \leq k \leq K-1,1 \leq j \leq J, \\
W_{j, 1}^{n+1}=U_{j, k}^{n}-2 \lambda^{y} \hat{g}\left(U_{j, 2}^{n}, U_{j, 1}^{n}\right), \quad 1 \leq j \leq J, \\
W_{j, K}^{n+1}=U_{j, k}^{n}+2 \lambda^{y} \hat{g}\left(U_{j, K}^{n}, U_{j, K-1}^{n}\right), \quad 1 \leq j \leq J . \\
U_{j, k}^{n+1}=(1 / 2) V_{j, k}^{n+1}+(1 / 2) W_{j, k}^{n+1} .
\end{array}\right.
\end{gathered}
$$

We will refer to (3.5) as the $x$ portion of the scheme, and 3.6 as the $y$ portion. It is clear that if we fix a single $k \in \mathbb{Z}_{K}$ in the $x$ portion, or if we fix $j \in \mathbb{Z}_{J}$ in the $y$ portion, we get a one-dimensional scheme for $u_{t}+f(u)_{x}=0$ in the first case, or $u_{t}+g(u)_{y}=0$ in the second case.

Remark 3.1. Instead of combining the $x$ and $y$ portions of the scheme via (3.7), we could also combine them via dimensional splitting. All of the results of this paper would remain true, and in fact the CFL condition below could be relaxed, resulting in twice the allowable time step $\Delta t$.

Remark 3.2. When $\left(x_{j}, y_{k}\right)$ and its neighboring gridpoints are away from the boundary of $\Omega$, the marching formulas (3.5), (3.6), (3.7) result in

$$
U_{j, k}^{n+1}=U_{j, k}^{n}-\lambda^{x} \Delta_{-}^{x} \hat{f}\left(U_{j+1, k}^{n}, U_{j, k}^{n}\right)-\lambda^{y} \Delta_{-}^{y} \hat{g}\left(U_{j, k+1}^{n}, U_{j, k}^{n}\right),
$$

as one might anticipate. In fact, we can extend 3.8 to the entire compuational grid, i.e., $(j, k) \in$ $\mathbb{Z}_{J} \times \mathbb{Z}_{K}$, if we agree that when a numerical flux involves an "out of bounds index " (meaning outside the range $1 \leq j \leq J, 1 \leq k \leq K)$, then we set that flux equal to zero. For example, we define $\hat{f}\left(U_{1, k}^{n}, U_{0, k}^{n}\right)=0$.

Remark 3.3. In the important one-dimensional case, where the spatial domain is $\Omega=\left[0, a_{1}\right]$, and the conservation law is $u_{t}+f(u)_{x}=0$, the scheme is simply

$$
\left\{\begin{array}{l}
U_{j}^{n+1}=U_{j}^{n}-\lambda^{x} \Delta_{-}^{x} \hat{f}\left(U_{j+1}^{n}, U_{j}^{n}\right), \quad 2 \leq j \leq J-1, \\
U_{1}^{n+1}=U_{1}^{n}-\lambda^{x} \hat{f}\left(U_{2}^{n}, U_{1}^{n}\right) \\
U_{J}^{n+1}=U_{J}^{n}+\lambda^{x} \hat{f}\left(U_{J}^{n}, U_{J-1}^{n}\right) .
\end{array}\right.
$$

\section{Convergence Analysis}

We will assume that the following CFL condition is satisfied:

$$
\begin{aligned}
& \text { For all } u, v, z, w \in[0,1], \\
& -\lambda^{x} \frac{\hat{f}(v, w)-\hat{f}(u, w)}{v-u}+\lambda^{x} \frac{\hat{f}(z, v)-\hat{f}(z, u)}{v-u} \leq \frac{1}{2}, \\
& -\lambda^{y} \frac{\hat{g}(v, w)-\hat{g}(u, w)}{v-u}+\lambda^{y} \frac{\hat{g}(z, v)-\hat{g}(z, u)}{v-u} \leq \frac{1}{2} .
\end{aligned}
$$

For the Godunov, Engquist-Osher, and Lax-Friedrichs (with $\alpha=1$ ) fluxes we can substitute the somewhat simpler CFL condition below, which implies 4.1):

$$
\lambda^{x} \max _{w \in[0,1]}\left|f^{\prime}(w)\right| \leq 1 / 2, \quad \lambda^{y} \max _{w \in[0,1]}\left|g^{\prime}(w)\right| \leq 1 / 2 .
$$


Having selected the spatial mesh sizes $\Delta x$ and $\Delta y$, the CFL condition amounts to a restriction on the size of the time step $\Delta t$. For the convergence analysis that follows, we will assume that the mesh size $\Delta \rightarrow 0$ with the CFL condition (4.1) satisfied.

Remark 4.1. For the one-dimensional scheme 3.9 , the $1 / 2$ on the right sides of the CFL conditions (4.1) and (4.2) can be replaced by 1 .

We will employ the following two measures of total variation of the numerical solution $U^{n}$ :

$$
\begin{aligned}
\operatorname{TV}\left(U^{n}\right) & :=\sum_{k=1}^{K} \Delta y \sum_{j=1}^{J-1}\left|\Delta_{+}^{x} U_{j, k}^{n}\right|+\sum_{j=1}^{J} \Delta x \sum_{k=1}^{K-1}\left|\Delta_{+}^{y} U_{j, k}^{n}\right|, \\
\operatorname{TV}^{*}\left(U^{n}\right) & :=\operatorname{TV}\left(U^{n}\right)+\sum_{k=1}^{K} \Delta y\left(U_{1, k}^{n}-U_{J, k}^{n}\right)+\sum_{j=1}^{J} \Delta x\left(U_{j, 1}^{n}-U_{j, K}^{n}\right) .
\end{aligned}
$$

The functional TV is the standard total variation for a grid function defined on a rectangle, while $\mathrm{TV}^{*}$ turns out to be a more convenient quantity for the zero-flux boundary value problem of this paper. Note that if $U_{j, k}^{n} \in[0,1]$ for all $(j, k) \in \mathbb{Z}_{J} \times \mathbb{Z}_{K}$ (which will be established in Lemma 4.1 below) then,

$$
\begin{aligned}
& \operatorname{TV}\left(U^{n}\right) \leq \operatorname{TV}^{*}\left(U^{n}\right)+a_{1}+a_{2}, \\
& \operatorname{TV}^{*}\left(U^{n}\right) \leq \operatorname{TV}\left(U^{n}\right)+a_{1}+a_{2} .
\end{aligned}
$$

It is readily verified that each of the $x$ and $y$ portions of the scheme, as well as the overall scheme, preserves total mass:

$$
\Delta x \Delta y \sum_{j=1}^{J} \sum_{k=1}^{K} U_{j, k}^{n+1}=\Delta x \Delta y \sum_{j=1}^{J} \sum_{k=1}^{K} V_{j, k}^{n+1}=\Delta x \Delta y \sum_{j=1}^{J} \sum_{k=1}^{K} W_{j, k}^{n+1}=\Delta x \Delta y \sum_{j=1}^{J} \sum_{k=1}^{K} U_{j, k}^{n} .
$$

The one-dimensional versions of the $x$ and $y$ portions are also total mass preserving (in the onedimensional sense):

$$
\begin{aligned}
& \Delta x \sum_{j=1}^{J} V_{j, k}^{n+1}=\Delta x \sum_{j=1}^{J} U_{j, k}^{n}, \quad k \in \mathbb{Z}_{K} \\
& \Delta y \sum_{k=1}^{K} W_{j, k}^{n+1}=\Delta y \sum_{k=1}^{K} U_{j, k}^{n}, \quad j \in \mathbb{Z}_{J} .
\end{aligned}
$$

Lemma 4.1. The scheme is monotone, meaning that

$$
\begin{aligned}
& \text { if } U_{j, k}^{n} \leq \tilde{U}_{j, k}^{n} \text { for all }(j, k) \in \mathbb{Z}_{J} \times \mathbb{Z}_{K}, \\
& \text { then } U_{j, k}^{n+1} \leq \tilde{U}_{j, k}^{n+1} \text { for all }(j, k) \in \mathbb{Z}_{J} \times \mathbb{Z}_{K} .
\end{aligned}
$$

In addition, the computed solution satisfies

$$
U_{j, k}^{n} \in[0,1], \quad \text { for all }(j, k) \in \mathbb{Z}_{J} \times \mathbb{Z}_{K}, \quad n=0,1,2, \ldots, N .
$$

Proof. Since $u_{0}(x, y) \in[0,1]$, due to 3.2 we will also have $U_{j, k}^{0} \in[0,1]$, and thus the CFL condition (4.1) will be satisfied when we compute $V^{1}$ and $W^{1}$. Consider the first equation in (3.5). It follows from a standard calculation for one-dimensional monotone schemes that for $2 \leq j \leq J-1,1 \leq k \leq K, V_{j, k}^{1}$ is a nondecreasing function of $U_{j-1, k}^{0}, U_{j, k}^{0}, U_{j+1, k}^{0}$. Now consider the second and third equations of (3.5). Using the CFL condition again, along with the monotonicity of the numerical flux, we find that $V_{1, k}^{1}$ is a nondecreasing function of $U_{1, k}^{0}$ and $U_{2, k}^{0}$, and that $V_{J, k}^{1}$ is a nondecreasing function of $U_{J-1, k}^{0}$ and $U_{J, k}^{0}$. Thus the $x$-portion of the scheme is monotone on the first time step, i.e.,

$$
\text { if } U_{j, k}^{0} \leq \tilde{U}_{j, k}^{0} \text { for all }(j, k) \in \mathbb{Z}_{J} \times \mathbb{Z}_{K} \text { then } V_{j, k}^{1} \leq \tilde{V}_{j, k}^{1} \text { for all }(j, k) \in \mathbb{Z}_{J} \times \mathbb{Z}_{K},
$$

and by a similar argument, the analogous monotonicity property holds for the $y$-portion of the scheme. Monotonicity of the overall scheme, (4.6), at least on the first time step, now follows from from the observation that the averaging step (3.7) preserves monotonicity. 
Now let Zero (One) denote the grid function that is identically $=0$ (identically $=1$ ). Both of these grid functions are fixed points of the scheme. The initial data satisfies

$$
\text { Zero }_{j, k} \leq U_{j, k}^{0} \leq \mathrm{One}_{j, k},
$$

and since we have established (4.6) for the first time step, we have

$$
\text { Zero }_{j, k} \leq U_{j, k}^{1} \leq \text { One }_{j, k} .
$$

Clearly, the foregoing argument can be repeated inductively, completing the proof.

Lemma 4.2. We have the following time continuity property for the computed solution:

$$
\Delta x \Delta y \sum_{j=1}^{J} \sum_{k=1}^{K}\left|U_{j, k}^{n+1}-U_{j, k}^{n}\right| \leq B \cdot \Delta t
$$

where $B$ is independent of $\Delta x, \Delta y, \Delta t$.

Proof. The monotonicity of the scheme, along with the total mass preserving property (4.4), allows us to apply the Crandall-Tartar lemma [17, which yields

$$
\Delta x \Delta y \sum_{j=1}^{J} \sum_{k=1}^{K}\left|U_{j, k}^{n+1}-U_{j, k}^{n}\right| \leq \Delta x \Delta y \sum_{j=1}^{J} \sum_{k=1}^{K}\left|U_{j, k}^{n}-U_{j, k}^{n-1}\right| \leq \ldots \leq \Delta x \Delta y \sum_{j=1}^{J} \sum_{k=1}^{K}\left|U_{j, k}^{1}-U_{j, k}^{0}\right| .
$$

It remains to estimate this last sum. From (3.5), we have, for fixed $k \in \mathbb{Z}_{K}$,

$$
\begin{aligned}
\sum_{j=1}^{J}\left|V_{j, k}^{1}-U_{j, k}^{0}\right| & \leq 2 \lambda^{x} \sum_{j=2}^{J-1}\left|\Delta_{-}^{x} \hat{f}\left(U_{j+1, k}^{0}, U_{j, k}^{0}\right)\right|+2 \lambda^{x}\left|\hat{f}\left(U_{2, k}^{0}, U_{1, k}^{0}\right)\right|+2 \lambda^{x}\left|\hat{f}\left(U_{J, k}^{0}, U_{J-1, k}^{0}\right)\right| \\
& \leq 4 \lambda^{x} L_{\bar{f}} \sum_{j=1}^{J-1}\left|\Delta_{+}^{x} U_{j, k}^{0}\right|+2 \lambda^{x}\left\|f\left(u_{0}\right)\right\|_{\infty} .
\end{aligned}
$$

Here $L_{\bar{f}}$ is a Lipschitz constant for the numerical flux $\bar{f}$ (and thus also for $\hat{f}$ ).

Multiplying (4.7) by $\Delta x \Delta y$, and then summing over $k$, the result is

$$
\Delta x \Delta y \sum_{k=1}^{K} \sum_{j=1}^{J}\left|V_{j, k}^{1}-U_{j, k}^{0}\right| \leq 4 \Delta x \lambda^{x} L_{\bar{f}} \sum_{k=1}^{K} \Delta y \sum_{j=1}^{J-1}\left|\Delta_{+}^{x} U_{j, k}^{0}\right|+2 a_{2} \Delta x \lambda^{x}\left\|f\left(u_{0}\right)\right\|_{\infty} .
$$

Replacing $\Delta x \lambda^{x}$ by $\Delta t$, and recalling that $u_{0} \in \mathrm{BV}(\Omega), u_{0}(x, y) \in[0,1]$ we have

$$
\Delta x \Delta y \sum_{k=1}^{K} \sum_{j=1}^{J}\left|V_{j, k}^{1}-U_{j, k}^{0}\right| \leq B_{1} \cdot \Delta t .
$$

A similar calculation starting from $(3.6)$ gives

$$
\Delta x \Delta y \sum_{j=1}^{J} \sum_{k=1}^{K}\left|W_{j, k}^{1}-U_{j, k}^{0}\right| \leq B_{2} \cdot \Delta t .
$$

Since

$$
\left|U_{j, k}^{1}-U_{j, k}^{0}\right| \leq(1 / 2)\left|V_{j, k}^{1}-U_{j, k}^{0}\right|+(1 / 2)\left|W_{j, k}^{1}-U_{j, k}^{0}\right|,
$$

we can combine 4.8 and 4.9:

$$
\Delta x \Delta y \sum_{k=1}^{K} \sum_{j=1}^{J}\left|U_{j, k}^{1}-U_{j, k}^{0}\right| \leq \frac{1}{2}\left(B_{1}+B_{2}\right) \Delta t,
$$

thus completing the proof. 
Lemma 4.3. The functional $T V^{*}$ is nonincreasing:

$$
T V^{*}\left(U^{n+1}\right) \leq T V^{*}\left(U^{n}\right), \quad n=0,1,2, \ldots, N,
$$

and we have the following bound on the total variation:

$$
T V\left(U^{n}\right) \leq T V\left(u_{0}\right)+2 a_{1}+2 a_{2}, \quad n=1,2, \ldots, N .
$$

Proof. Consider the $x$ portion of the scheme (3.5) for a fixed $k \in \mathbb{Z}_{K}$. Since the index $k$ is fixed for the moment, we simplify the notation by temporarily omitting it. This one-dimensional $x$-portion of the scheme 3.5 can be written in incremental form:

$$
\begin{aligned}
V_{j}^{n+1} & =U_{j}^{n}+C_{j+\frac{1}{2}}^{n} \Delta_{+}^{x} U_{j}^{n}-D_{j-\frac{1}{2}}^{n} \Delta_{-}^{x} U_{j}^{n}, \quad 2 \leq j \leq J-1, \\
V_{1}^{n+1} & =U_{1}^{n}-2 \lambda^{x} \hat{f}\left(U_{2}^{n}, U_{1}^{n}\right) \\
V_{J}^{n+1} & =U_{J}^{n}+2 \lambda^{x} \hat{f}\left(U_{J}^{n}, U_{J-1}^{n}\right)
\end{aligned}
$$

where

$$
C_{j+\frac{1}{2}}^{n}=-2 \lambda^{x} \frac{\hat{f}\left(U_{j+1}^{n}, U_{j}^{n}\right)-f\left(U_{j}^{n}\right)}{\Delta_{+}^{x} U_{j}^{n}}, \quad D_{j-\frac{1}{2}}^{n}=2 \lambda^{x} \frac{f\left(U_{j}^{n}\right)-\hat{f}\left(U_{j}^{n}, U_{j-1}^{n}\right)}{\Delta_{-}^{x} U_{j}^{n}} .
$$

Due the fact that the numerical flux $\hat{f}$ is monotone, and to the CFL condition 4.1], the incremental coefficients satisfy

$$
C_{j+\frac{1}{2}}^{n} \geq 0, \quad D_{j+\frac{1}{2}}^{n} \geq 0, \quad C_{j+\frac{1}{2}}^{n}+D_{j+\frac{1}{2}}^{n} \leq 1 .
$$

An application of $\Delta_{+}^{x}$ to 4.10 results in

$$
\begin{aligned}
& \Delta_{+}^{x} V_{j}^{n+1}=\left(1-C_{j+\frac{1}{2}}^{n}-D_{j+\frac{1}{2}}^{n}\right) \Delta_{+}^{x} U_{j}^{n}+C_{j+\frac{3}{2}}^{n} \Delta_{+}^{x} U_{j+1}^{n}+D_{j-\frac{1}{2}}^{n} \Delta_{+}^{x} U_{j-1}^{n}, \quad 2 \leq j \leq J-2, \\
& \Delta_{+}^{x} V_{1}^{n+1}=\left(1-D_{\frac{3}{2}}^{n}\right) \Delta_{+}^{x} U_{1}^{n}+C_{\frac{5}{2}}^{n} \Delta_{+}^{x} U_{2}^{n}+2 \lambda^{x} \hat{f}\left(U_{2}^{n}, U_{1}^{n}\right), \\
& \Delta_{+}^{x} V_{J-1}^{n+1}=\left(1-C_{J-\frac{1}{2}}^{n}\right) \Delta_{+}^{x} U_{J-1}^{n}+D_{J-\frac{3}{2}}^{n} \Delta_{+}^{x} U_{J-2}^{n}+2 \lambda^{x} \hat{f}\left(U_{J}^{n}, U_{J-1}^{n}\right) .
\end{aligned}
$$

Due to 4.11, and the fact that $\hat{f} \geq 0$, it follows from 4.12 that

$$
\begin{aligned}
\left|\Delta_{+}^{x} V_{j}^{n+1}\right| \leq & \left(1-C_{j+\frac{1}{2}}^{n}-D_{j+\frac{1}{2}}^{n}\right)\left|\Delta_{+}^{x} U_{j}^{n}\right| \\
& \quad+C_{j+\frac{3}{2}}^{n}\left|\Delta_{+}^{x} U_{j+1}^{n}\right|+D_{j-\frac{1}{2}}^{n}\left|\Delta_{+}^{x} U_{j-1}^{n}\right|, \quad 1 \leq j \leq J-2, \\
\left|\Delta_{+}^{x} V_{1}^{n+1}\right| \leq & \left(1-D_{\frac{3}{2}}^{n}\right)\left|\Delta_{+}^{x} U_{1}^{n}\right|+C_{\frac{5}{2}}^{n}\left|\Delta_{+}^{x} U_{2}^{n}\right|+2 \lambda^{x} \hat{f}\left(U_{2}^{n}, U_{1}^{n}\right) \\
\left|\Delta_{+}^{x} V_{J-1}^{n+1}\right| \leq & \left(1-C_{J-\frac{1}{2}}^{n}\right)\left|\Delta_{+}^{x} U_{J-1}^{n}\right|+D_{J-\frac{3}{2}}^{n}\left|\Delta_{+}^{x} U_{J-2}^{n}\right|+2 \lambda^{x} \hat{f}\left(U_{J}^{n}, U_{J-1}^{n}\right) .
\end{aligned}
$$

Summing over $1 \leq j \leq J-1$, and then canceling the telescoping terms, we get

$$
\left.\sum_{j=1}^{J-1}\left|\Delta_{+}^{x} V_{j}^{n+1}\right| \leq \sum_{j=1}^{J-1}\left|\Delta_{+}^{x} U_{j}^{n}\right|+2 \lambda^{x} \hat{f}\left(U_{2}^{n}, U_{1}^{n}\right)\right)+2 \lambda^{x} \hat{f}\left(U_{J}^{n}, U_{J-1}^{n}\right) .
$$

After substituting

$$
\left.2 \lambda^{x} \hat{f}\left(U_{2}^{n}, U_{1}^{n}\right)\right)=U_{1}^{n}-V_{1}^{n+1}, \quad 2 \lambda^{x} \hat{f}\left(U_{J}^{n}, U_{J-1}^{n}\right)=V_{J}^{n+1}-U_{J}^{n},
$$

and then rearranging, the result is

$$
\sum_{j=1}^{J-1}\left|\Delta_{+}^{x} V_{j}^{n+1}\right|+V_{1}^{n+1}-V_{J}^{n+1} \leq \sum_{j=1}^{J-1}\left|\Delta_{+}^{x} U_{j}^{n}\right|+U_{1}^{n}-U_{J}^{n} .
$$

At this point, we reinstate the $k$ part of the subscript. Multiplying 4.13 by $\Delta y$, and then summing over $k$, the result is

$$
\sum_{k=1}^{K} \Delta y\left(\sum_{j=1}^{J-1}\left|\Delta_{+}^{x} V_{j, k}^{n+1}\right|+V_{1, k}^{n+1}-V_{J, k}^{n+1}\right) \leq \sum_{k=1}^{K} \Delta y\left(\sum_{j=1}^{J-1}\left|\Delta_{+}^{x} U_{j, k}^{n}\right|+U_{1, k}^{n}-U_{J, k}^{n}\right) .
$$

Still focusing on the $x$ portion of the scheme, recall the first equation of 4.5 , which states that the one-dimensional version (which results when $k$ is fixed) preserves total mass. Also, the proof 
of Lemma 4.1 reveals that the one-dimensional version of the $x$ portion is monotone. Thus, we can apply the Crandall-Tartar lemma [17]:

$$
\Delta x \sum_{j=1}^{J}\left|V_{j, k+1}^{n+1}-V_{j, k}^{n+1}\right| \leq \Delta x \sum_{j=1}^{J}\left|U_{j, k+1}^{n}-U_{j, k}^{n}\right|, \quad k=1, \ldots, K-1 .
$$

Applying the first equation of 4.5, we find that

$$
\sum_{j=1}^{J} \Delta x V_{j, 1}^{n+1}=\sum_{j=1}^{J} \Delta x U_{j, 1}^{n}, \quad \sum_{j=1}^{J} \Delta x V_{j, K}^{n+1}=\sum_{j=1}^{J} \Delta x U_{j, K}^{n},
$$

which we combine as follows:

$$
\sum_{j=1}^{J} \Delta x\left(V_{j, 1}^{n+1}-V_{j, K}^{n+1}\right)=\sum_{j=1}^{J} \Delta x\left(U_{j, 1}^{n}-U_{j, K}^{n}\right) .
$$

Summing 4.15) over $k$, and then adding (4.16), we get

$$
\begin{aligned}
& \sum_{k=1}^{K-1} \Delta x \sum_{j=1}^{J} \mid V_{j, k+1}^{n+1}-V_{j, k}^{n+1} \mid+\sum_{j=1}^{J} \Delta x\left(V_{j, 1}^{n+1}-V_{j, K}^{n+1}\right) \\
& \leq \sum_{k=1}^{K-1} \Delta x \sum_{j=1}^{J}\left|U_{j, k+1}^{n}-U_{j, k}^{n}\right|+\sum_{j=1}^{J} \Delta x\left(U_{j, 1}^{n}-U_{j, K}^{n}\right) .
\end{aligned}
$$

Adding (4.14) to 4.17), we arrive at

$$
T V^{*}\left(V^{n+1}\right) \leq T V^{*}\left(U^{n}\right) .
$$

The same type of calculation, this time applied to the $y$ portion (3.6), yields

$$
T V^{*}\left(W^{n+1}\right) \leq T V^{*}\left(U^{n}\right) .
$$

It is readily verified that the $\mathrm{TV}^{*}$ functional is convex, and so

$$
\begin{aligned}
T V^{*}\left(U^{n+1}\right) & =T V^{*}\left((1 / 2) V^{n+1}+(1 / 2) W^{n+1}\right) \\
& \leq(1 / 2) \mathrm{TV}^{*}\left(V^{n+1}\right)+(1 / 2) \mathrm{TV}^{*}\left(W^{n+1}\right) \\
& \leq T V^{*}\left(U^{n}\right) .
\end{aligned}
$$

By induction on $n$, we thus have that for all $n=0,1, \ldots, N$,

$$
\begin{aligned}
T V^{*}\left(U^{n}\right) \leq \mathrm{TV}^{*}\left(U^{0}\right) & \leq \mathrm{TV}\left(U^{0}\right)+a_{1}+a_{2} \\
& \leq \mathrm{TV}\left(u_{0}\right)+a_{1}+a_{2} .
\end{aligned}
$$

Here we have used the second inequality of 4.3 . Using the first inequality of 4.3 , we have

$$
\mathrm{TV}\left(U^{n}\right) \leq \mathrm{TV}\left(u_{0}\right)+2 a_{1}+2 a_{2} .
$$

Lemmas 4.1 , 4.2 and 4.3 give an $L^{\infty}$ bound, a bound on $L^{1}$ time translates, and a spatial variation bound. With these bounds, the following lemma results from a standard compactness argument [1] and the Lax-Wendroff theorem.

Lemma 4.4. The sequence of approximations $u^{\Delta}$ converges along a subsequence in $L^{1}\left(Q_{T}\right)$ and boundedly a.e. to a function $u \in L^{1}\left(Q_{T}\right) \cap B V\left(Q_{T}\right)$. The limit function $u$ is a weak solution of the conservation law $u_{t}+f(u)_{x}+g(u)_{y}=0$ in $Q_{T}$.

Remark 4.2. We will see below (Theorem 4.1 that the convergence of $u^{\Delta}$ is actually not just along a subsequence. The entire computed sequence converges. 


\begin{tabular}{l|l|l}
\multicolumn{1}{c|}{$\Omega_{\mathrm{nw}}^{\mathrm{c}}$} & $\Omega_{\mathrm{n}}^{\mathrm{c}}$ & $\Omega_{\mathrm{ne}}^{\mathrm{c}}$ \\
$\mathrm{v}_{0}=0$ or 1 & $\mathrm{v}_{0}=1$ & $\mathrm{v}_{0}=1$ \\
\cline { 2 - 3 }$\Omega_{\mathrm{w}}^{\mathrm{c}}$ & $\Omega_{0}$ & $\Omega_{\mathrm{e}}^{\mathrm{c}}$ \\
\hline $\mathrm{v}_{0}=0$ & $\mathrm{v}_{0}=\mathrm{u}_{0}$ & $\mathrm{v}_{0}=1$ \\
\hline$\Omega_{\mathrm{sw}}^{\mathrm{c}}$ & $\Omega_{\mathrm{s}}^{\mathrm{c}}$ & $\Omega_{\mathrm{se}}^{\mathrm{c}}$ \\
$\mathrm{v}_{0}=0$ & $\mathrm{v}_{0}=0$ & $\mathrm{v}_{0}=0$ or 1
\end{tabular}

Figure 1. Extension of initial data from $\Omega$ to $\mathbb{R}^{2}$. With the data extended in this way, the numerical fluxes evaluate to zero outside of $\Omega$, due to the fact that $\hat{f}(0,0)=\hat{f}(0,1)=\hat{f}(1,1)=0$, and similarly for $\hat{g}$.

Next, we set out to verify that the limit $u$ of the numerical scheme is an entropy solution in the sense of Definition 2.1. We start by extending the initial data, and the scheme, to all of $\mathbb{R}^{2}$. The spatial grid is extended in the obvious way, taking $j \in \mathbb{Z}, k \in \mathbb{Z}$ in (3.1). The extended initial data is denoted $v_{0}(x, y)$, and the extended numerical solution is denoted $V_{j, k}^{n}$. There are several ways of extending the initial data that are suitable for our purposes; see Figure 1. For the sake of concreteness, we extend $u_{0}$ according to

$$
v_{0}(x, y)= \begin{cases}u_{0}(x, y), \quad(x, y) \in \Omega \\ 0, \quad x \leq 0 \\ 1, \quad x \geq 1 \\ 0, \quad y \leq 0, x \in\left(0, a_{1}\right) \\ 1, \quad y \geq 1, x \in\left(0, a_{1}\right) .\end{cases}
$$

Referring to Figure 1, this corresponds to taking $v_{0}(x, y)=0$ in $\Omega_{n w}^{c}$, and $v_{0}(x, y)=1$ in $\Omega_{s e}^{c}$. The extended scheme is then exactly formula (3.8) of Remark 3.2 , except that now we ignore the requirement to set certain numerical fluxes equal to zero if they have indices that are out of bounds. We define the discrete initial data $V_{j, k}^{0}$ and the approximate solution $v^{\Delta}(x, y, t)$ by extending the formulas (3.2) and (3.3) in the obvious way. In fact, based on (4.18), the discrete version of $v_{0}$ will be

$$
V_{j, k}^{0}= \begin{cases}U_{j, k}^{0}, \quad(j, k) \in \mathbb{Z}_{J} \times \mathbb{Z}_{k} \\ 0, \quad j<1 \\ 1, \quad j>J \\ 0, \quad k<1, \quad 1 \leq j \leq J \\ 1, \quad k>K, \quad 1 \leq j \leq J .\end{cases}
$$

For the type of fluxes $q(u)$ appearing in this paper, i.e., $q(0)=q(1)=0, q(u) \geq 0$, the modified numerical flux has the following property for $u, v \in[0,1]$, which plays a crucial role in what follows:

$$
\hat{q}(v, 0)=0, \quad \hat{q}(1, u)=0 .
$$


To see this, note that by monotonicity and consistency we have

$$
\begin{aligned}
& \hat{q}(v, 0) \leq \hat{q}(0,0)=0, \\
& \hat{q}(1, u) \leq \hat{q}(1,1)=0 .
\end{aligned}
$$

Lemma 4.5. For mesh points $\left(x_{j}, y_{k}\right) \in \Omega$ (equivalently $\left.(j, k) \in \mathbb{Z}_{J} \times \mathbb{Z}_{K}\right)$, the extended numerical solution agrees with the original numerical solution, i.e.,

$$
V_{j, k}^{n}=U_{j, k}^{n}, \quad(j, k) \in \mathbb{Z}_{J} \times \mathbb{Z}_{K}, \quad n=0,1,2, \ldots, N .
$$

and thus $v^{\Delta}(x, y, t)=u^{\Delta}(x, y, t)$ for $(x, y) \in \Omega$.

Proof. Clearly, $V_{j, k}^{0}=U_{j, k}^{0}$ for $\left(x_{j}, y_{k}\right) \in \Omega$. Given a grid point $\left(x_{j}, y_{k}\right)$ we define its neighbors to be the four grid points $\left(x_{j \pm 1}, y_{k}\right),\left(x_{j}, y_{k \pm 1}\right)$. Due to the stencil of the scheme, it is clear that if $\left(x_{j}, y_{k}\right) \in \Omega$, and if all four of its neighbors are also in $\Omega$ then $V_{j, k}^{1}=U_{j, k}^{1}$.

We wish to show that $V_{j, k}^{1}=U_{j, k}^{1}$ in the remaining cases also. Before proceeding, consider a numerical flux $\hat{q}(v, u)$ where one or both of the arguments correspond to a point $\left(x_{j}, y_{k}\right) \notin \Omega$. Due to the way that we extended the initial data, such a flux is of the form $\hat{q}(v, 0)$ or $\hat{q}(1, u)$ where $q=f$ or $q=g$. According to 4.19 , each such numerical flux vanishes.

Now suppose that $\left(x_{j}, y_{k}\right) \in \Omega$, but one or more of its neighbors lies outside of $\Omega$. Then $U_{j, k}^{1}$ is computed using (3.8), setting any numerical flux with an out of bounds index to zero. But according to the observation above, the extended scheme will set those same numerical fluxes equal to zero, and so we will have $V_{j, k}^{1}=U_{j, k}^{1}$ in this case also.

We claim that for gridpoints $\left(x_{j}, y_{k}\right) \notin \Omega$, we will also have $V_{j, k}^{1}=V_{j, k}^{0}$. All numerical fluxes involved in this case have at least one argument corresponding to a point $\left(x_{j}, y_{k}\right) \notin \Omega$. All such numerical fluxes vanish, and so $V_{j, k}^{1}=V_{j, k}^{0}$.

We have shown 4.20 holds for $n=1$. It is clear that we can repeat this argument at the next time level, completing the proof by induction on $n$.

Recall the Kružkov entropy function $|u-\kappa|$, indexed by $\kappa \in \mathbb{R}$, and the associated entropy fluxes $F(u)=\operatorname{sign}(u-\kappa)(f(u)-f(\kappa)), G(u)=\operatorname{sign}(u-\kappa)(g(u)-g(\kappa))$. Following [11, we define the numerical entropy fluxes:

$$
\begin{aligned}
& \hat{F}(v, u):=\hat{f}(v \vee \kappa, u \vee \kappa)-\hat{f}(v \wedge \kappa, u \wedge \kappa), \\
& \hat{G}(v, u):=\hat{g}(v \vee \kappa, u \vee \kappa)-\hat{g}(v \wedge \kappa, u \wedge \kappa) .
\end{aligned}
$$

Here, and in what follows, we suppress the dependence on $\kappa$, in order to simplify the notation. The numerical entropy fluxes $\hat{F}$ and $\hat{G}$ are consistent with $F$ and $G$, i.e., $\hat{F}(u, u)=F(u)$ and $\hat{G}(u, u)=G(u)$.

Lemma 4.6. For the extended scheme, we have the following discrete entropy inequalities (one for each $\kappa \in \mathbb{R})$ :

$$
V_{j, k}^{n+1} \leq V_{j, k}^{n}-\lambda^{x} \Delta_{-}^{x} \hat{F}\left(V_{j+1, k}^{n}, V_{j, k}^{n}\right)-\lambda^{y} \Delta_{-}^{y} \hat{G}\left(V_{j, k+1}^{n}, V_{j, k}^{n}\right), \quad(j, k) \in \mathbb{Z} \times \mathbb{Z} .
$$

Proof. With the scheme extended in this way, the proof of this lemma is standard as in [11].

Lemma 4.7. The sequence of approximations $v^{\Delta}$ converges along a subsequence in $L_{\mathrm{loc}}^{1}\left(\Pi_{T}\right)$ and boundedly a.e. to a function $v \in L_{\mathrm{loc}}^{1}\left(\Pi_{T}\right) \cap B V_{\mathrm{loc}}\left(\Pi_{T}\right)$. The limit function $v$ is a weak solution of the conservation law $v_{t}+f(v)_{x}+g(v)_{y}=0$ in $\Pi_{T}$.

Proof. By construction, $V_{j, k}^{n} \in[0,1]$. Also by construction,

$$
\Delta x \Delta y \sum_{j \in \mathbb{Z}} \sum_{k \in \mathbb{Z}}\left|V_{j, k}^{n+1}-V_{j, k}^{n}\right|=\Delta x \Delta y \sum_{j=1}^{J} \sum_{k=1}^{K}\left|U_{j, k}^{n+1}-U_{j, k}^{n}\right| \leq B \cdot \Delta t,
$$

with the same constant $B$ appearing in the statement of Lemma 4.2 .

In addition, we claim that the spatial total variation of $v^{\Delta}$ is locally bounded. Let $\mathcal{K}$ be a compact subset of $\mathbb{R}^{2}$. Fix a time level $t^{n}$. Let $v^{\Delta}\left(t^{n}\right):=v^{\Delta}\left(\cdot, \cdot, t^{n}\right)$. Then

$$
\operatorname{TV}_{\mathcal{K}}\left(v^{\Delta}\left(t^{n}\right)\right) \leq \operatorname{TV}_{\mathcal{K} \cap \Omega}\left(v^{\Delta}\left(t^{n}\right)\right)+\operatorname{TV}_{\mathcal{K} \cap \Omega^{c}}\left(v^{\Delta}\left(t^{n}\right)\right)+2 a_{1}+2 a_{2},
$$


where $\Omega^{c}$ denotes the complement of $\Omega$. Since $v^{\Delta}=u^{\Delta}$ for $(x, y) \in \Omega$, Lemma 4.3 provides a uniform bound on $\operatorname{TV}_{\mathcal{K} \cap \Omega}\left(v^{\Delta}\left(t^{n}\right)\right)$. By construction, the function $v^{\Delta}\left(t^{n}\right)$ is constant in $\mathcal{K} \cap \Omega^{c}$, except for unit jumps along two half-lines. Thus $\operatorname{TV}_{\mathcal{K} \cap \Omega^{c}}\left(v^{\Delta}\left(t^{n}\right)\right)$ is also uniformly bounded.

With these bounds, the proof is concluded exactly like the proof of Lemma 4.4 .

By extracting further subsequences, we can assume in what follows that $u^{\Delta} \rightarrow u$ and $v^{\Delta} \rightarrow v$ along a common subsequence, so that we will have $\left.v\right|_{Q_{T}}=u$.

Lemma 4.8. The limit solution $u$ is an entropy solution in the sense of Definition 2.1.

Proof. Starting from (4.22), by a standard Lax-Wendroff type calculation (see eg., [11), we find that for any $\kappa \in \mathbb{R}$ and any test function $\phi \in C_{0}^{\infty}\left(\underline{\Pi}_{T}\right)$ with $\phi \geq 0$,

$$
\begin{aligned}
\int_{0}^{T} \int_{\mathbb{R}^{2}}\{\mid & \left.-\kappa \mid \phi_{t}+\operatorname{sign}(v-\kappa)(f(v)-f(\kappa)) \phi_{x}+\operatorname{sign}(v-\kappa)(g(v)-g(\kappa)) \phi_{y}\right\} d x d y d t \\
& +\int_{0}^{T} \int_{\mathbb{R}^{2}}\left|v_{0}(x, y)-\kappa\right| \phi(x, y, 0) d x d y \geq 0 .
\end{aligned}
$$

With the observation that $C_{0}^{\infty}\left(Q_{T}\right) \subset C_{0}^{\infty}\left(\underline{\Pi}_{T}\right)$, and that $v=u$ on $Q_{T}$, the inequality 4.23) implies that the entropy inequality (2.1) of Definition (2.1) is satisfied for our limit solution $u$. Moreover, the initial condition (2.2) follows easily from (4.23) and the BV regularity of $u$.

It remains to prove the limit satisfies the boundary condition 2.3). Starting from the extended difference scheme, again by a standard Lax-Wendroff type computation, we conclude that for any test function $\phi \in C_{0}^{\infty}\left(\Pi_{T}\right)$,

$$
\int_{0}^{T} \int_{\mathbb{R}^{2}}\left\{v \phi_{t}+f(v) \phi_{x}+g(v) \phi_{y}\right\} d x d y d t=0 .
$$

Since $v \in \operatorname{BV}\left(Q_{T}\right)$, which ensures the existence of a strong trace $v^{\tau}$ on $\partial \Omega \times(0, T)$, it follows from (4.24) that

$$
\left(f\left(v^{\tau}\right), g\left(v^{\tau}\right)\right) \cdot \nu=0 \text { a.e. on } \partial \Omega \times(0, T) .
$$

Clearly, $u=v$ on $Q_{T}$ implies that $v^{\tau}=u^{\tau}$, and hence the boundary condition (2.3) follows.

Theorem 4.1. Suppose the flux functions $f, g$ are Lipschitz-continuous and satisfy, for $h=f, g$, $h(0)=h(1)=0, h \geq 0$ on $[0,1]$. Moreover, suppose $u_{0}(x) \in[0,1]$, and that $u_{0} \in B V(\Omega)$. Then the approximate solutions $\left\{u^{\Delta}\right\}_{\Delta>0}$, defined by (3.3), 3.5), 3.6), and (3.7), converge in $L^{1}\left(Q_{T}\right)$ to the unique entropy solution $u$ of the initial-boundary value problem

$$
\left\{\begin{array}{l}
u_{t}+f(u)_{x}+g(u)_{y}=0 \quad \text { in } Q_{T}, \\
(f(u), g(u)) \cdot \nu=0 \quad \text { on } \partial \Omega \times(0, T), \\
u=u_{0} \quad \text { on } \Omega \times\{t=0\},
\end{array}\right.
$$

where $\Omega \subset \mathbb{R}^{2}$ is an open rectangular region.

Proof. By Lemma $4.4\left\{u^{\Delta}\right\}_{\Delta>0}$ converges along a subsequence. Recalling Lemma 4.8 the limit $u$ of any such convergent subsequence is an entropy solution in the sense of Definition 2.1. The uniqueness claim follows from Theorem 2.1. Moreover, since entropy solutions are unique, the convergence of $u^{\Delta}$ is not just along a subsequence, but along the entire computed sequence.

\section{NuMERICAL EXAMPLES}

Example 1. Figure 2 shows a one-dimensional example. The spatial domain is $\Omega=(0,1)$, and the flux is $f(u)=u(1-u)$. The initial data is

$$
u_{0}(x)= \begin{cases}1, & x \leq 1 / 2, \\ 0, & x>1 / 2 .\end{cases}
$$

We apply the one-dimensional version of the scheme, using the Godunov flux and the (modified) Lax-Friedrichs flux, with $\alpha=1$. The mesh size is $(\Delta x, \Delta t)=(.02, .015)$. The Lax-Friedrichs approximation is more diffusive than the Godunov approximation, as expected. The solution 

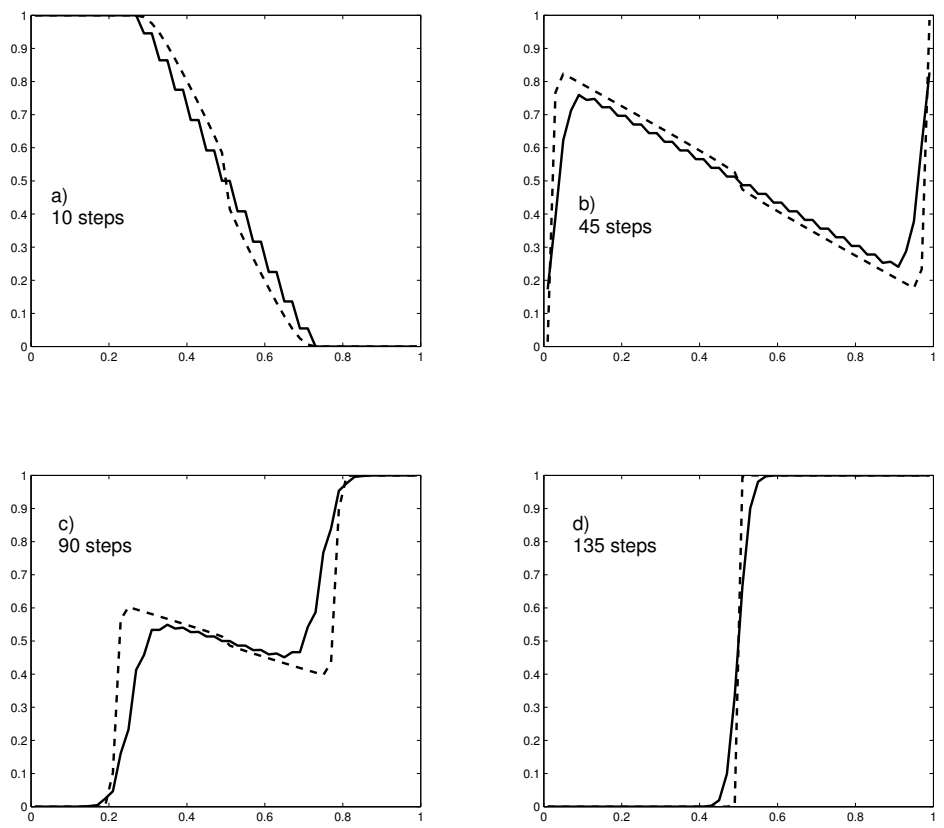

Figure 2. Example 1. The solid line is the (modified) Lax-Friedrichs solution, and the dashed line is the Godunov solution. Plot a) shows the development of a rarefaction fan. In Plot $b$ ), the edges of the rarefaction fan have reached the boundary, and shocks have formed. Plot c) shows the two shocks moving toward each other. In Plot d), a single steady shock remains.

initially develops a (decreasing) rarefaction fan. When the edges of the rarefaction reach the boundaries, the solution at the left (right) boundary begins to decrease (increase) as a function of time, and we get a pair of (increasing) shocks, one moving right, the other moving left. These shocks are visible in panel c) of Figure 2. Eventually, the two shocks meet, the rarefaction is annihilated by the shocks, and only a single steady shock remains.

This problem corresponds to a simple traffic flow model, with cars traveling from left to right. The zero flux boundary conditions model a pair of red lights, so that cars can neither enter nor leave this road segment. We start with all of the cars on the left half of the road, and none on the right half. With all of the cars moving to the right, we end up with the situation reversed. The steady shock that eventually develops models a traffic jam, with no movement of cars, as one might expect.

Example 2. Figure 3 shows a two-dimensional example using the Godunov flux. The spatial domain is $\Omega=(0,1) \times(0,1)$, and we take $f(u)=g(u)=u(1-u)$. Figure 4 shows the same problem, but using the modified Lax-Friedrichs flux. The mesh size is $(\Delta x, \Delta y, \Delta t)=(.02, .02, .009)$. The initial data is

$$
u_{0}(x)= \begin{cases}\cos (.5 \pi r), & .5 \pi r \leq .75 \\ 0, & .5 \pi r>.75\end{cases}
$$

where $r=\sqrt{1.5(x-.25)^{2}+.6(y-.25)^{2}}$. We start with all of the mass concentrated near the $(0,0)$ corner of $\Omega$. With increasing time the mass is convected toward the $(1,1)$ corner, eventually reaching a steady state. The steady state solution features an increasing jump from $u=0$ to $u=1$. As expected the Lax-Friedrichs solution is more diffusive than the Godunov solution. 

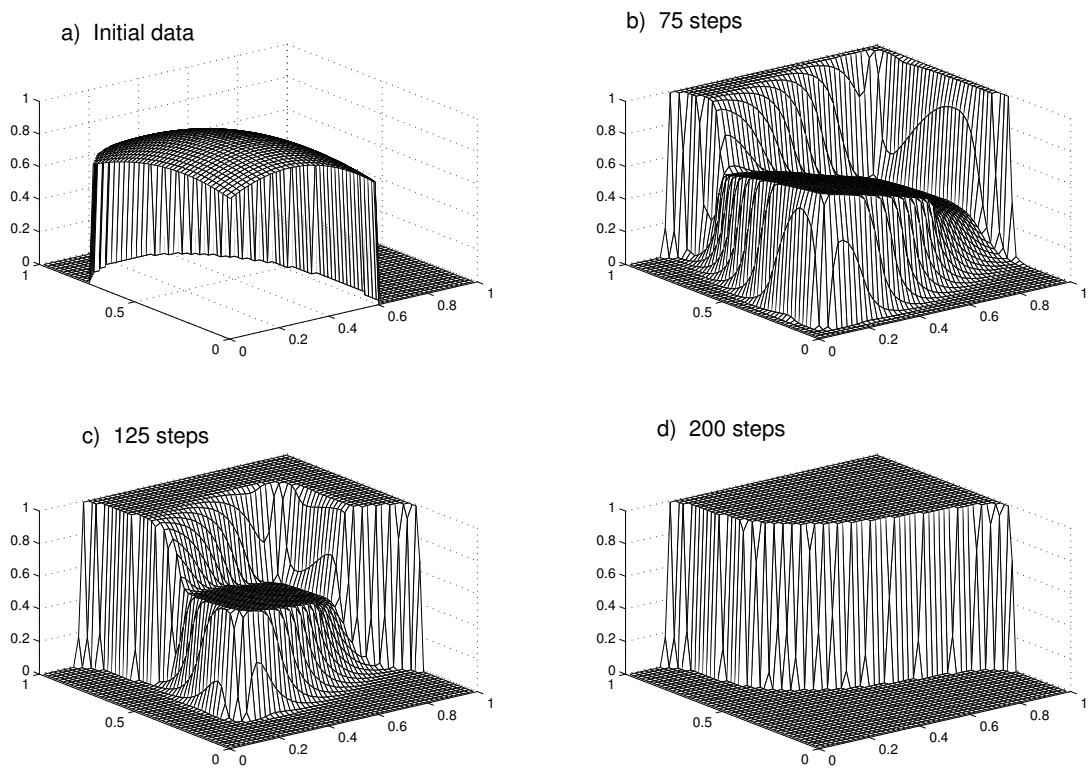

Figure 3. Example 2. Godunov flux. Solution shown in plot d) is essentially steady state.

\section{Degenerate CONVECtion-Diffusion EQUations}

Closely related to $(1.1)$ is the zero-flux initial boundary value problem for a degenerate convectiondiffusion equation, which we state as follows:

$$
\left\{\begin{array}{l}
u_{t}+\nabla \cdot f(u)-\Delta A(u)=0, \quad\left(x_{1}, \ldots, x_{d}\right) \in \Omega, \quad t \in(0, T), \\
(f(u(x, t))-\nabla A(u)) \cdot \nu=0 \text { a.e. on } \partial \Omega \times(0, T), \\
u(x, 0)=u_{0}(x), \quad x \in \Omega,
\end{array}\right.
$$

where the convective flux $f$ is as before. Regarding the diffusion function $A$, we assume that it belongs to $\operatorname{Lip}([0,1])$, with Lipschitz constant $\left\|A^{\prime}\right\|$, and that the following degenerate parabolicity condition holds:

$$
A(\cdot) \text { is nondecreasing with } A(0)=0 .
$$

We allow for the situation where $A$ degenerates (i.e., is constant) on a finite set of disjoint intervals, that is,

$$
A^{\prime}(w)=0, \quad \forall w \in \bigcup_{i=1}^{M}\left[\alpha_{i}, \beta_{i}\right],
$$

where $\alpha_{i}<\beta_{i}, i=1, \ldots, M, M \geq 1$. With this condition, the problem is said to be strongly degenerate. A significant consequence of strong degeneracy is that solutions are not generally continuous, and there is no uniqueness without an additional entropy condition. An important application where the problem (6.1) arises is pressure filtration of a flocculated suspension [6].

In [1] Andreianov and Gazibo define a notion of entropy solution for (6.1), and prove existence of entropy solutions using a vanishing viscosity approximation. They prove uniqueness of entropy solutions in the one-dimensional case. In 14 Gazibo proposes and analyzes an implicit finite volume scheme for (6.1). Andreianov and Gazibo prove convergence of that scheme to an entropy solution in 2, 14.

The degenerate parabolic problem (6.1) exhibits some difficulties that are not present in the purely hyperbolic problem (1.1). For the purely hyperbolic problem, our spatial variation bound guarantees strong traces of $u$ and therefore also strong traces of the normal component of the flux 
a) Initial data

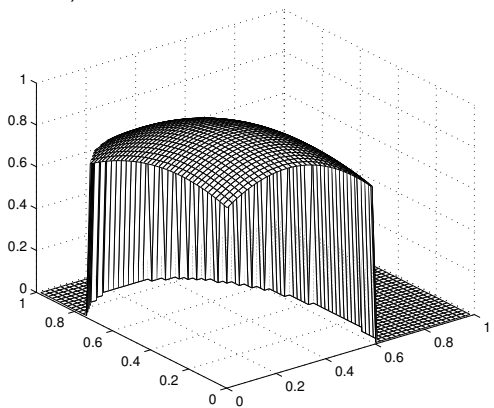

c) 125 steps

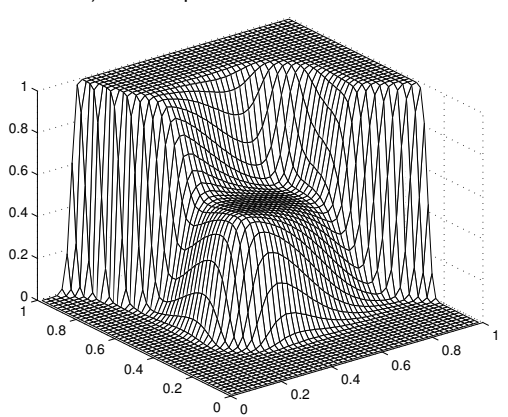

b) 75 steps

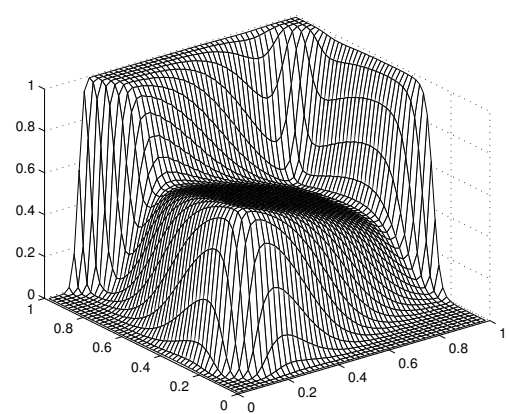

d) 200 steps

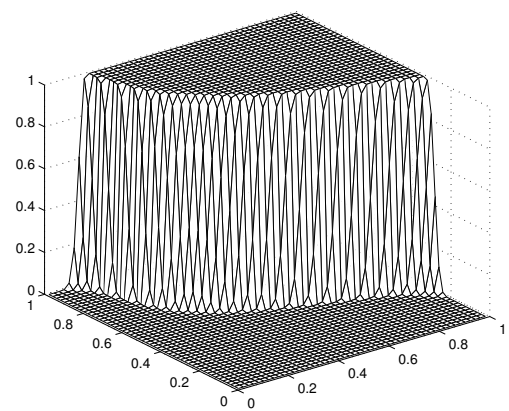

Figure 4. Example 2. Lax-Friedrichs flux. Solution shown in plot d) is essentially steady state.

$(f(u), g(u)) \cdot \nu$. For the degenerate parabolic problem, one would like to have strong traces of the total flux $\left(f(u)-A(u)_{x}, g(u)-A(u)_{y}\right) \cdot \nu$. This would follow from a spatial variation bound for $A(u)_{x}$ and $A(u)_{y}$, but such a bound is not available. However, by specializing to the onedimensional problem, we obtain a spatial variation bound for the total flux $f(u)-A(u)_{x}$, and so we do have the required strong trace in this important case, see Remark 6.4 below.

In what follows, we extend our scheme to handle the problem (6.1), and discuss some results that are straightforward extensions of those in Section 4 . Due to the difficulties mentioned in the previous paragraph, our results for the multidimensional problem are less complete than for the purely hyperbolic problem. We then focus on the one-dimensional problem, and prove convergence to the unique entropy solution.

The difference scheme discussed in the previous sections is easily modified to incorporate the diffusion term. With $\mu^{x}(\Delta)=\Delta t / \Delta x^{2}, \mu^{y}(\Delta)=\Delta t / \Delta y^{2}$, the difference equations (3.5), (3.6), (3.7) become:

$$
\begin{aligned}
& \left\{\begin{array}{l}
V_{j, k}^{n+1}=U_{j, k}^{n}-2 \lambda^{x} \Delta_{-}^{x} \hat{f}\left(U_{j+1, k}^{n}, U_{j, k}^{n}\right)+2 \mu^{x} \Delta_{+}^{x} \Delta_{-}^{x} A_{j, k}^{n}, \quad 2 \leq j \leq J-1,1 \leq k \leq K, \\
V_{1, k}^{n+1}=U_{1, k}^{n}-2 \lambda^{x} \hat{f}\left(U_{2, k}^{n}, U_{1, k}^{n}\right)+2 \mu^{x} \Delta_{+}^{x} A_{1, k}^{n}, \quad 1 \leq k \leq K, \\
V_{J, k}^{n+1}=U_{J, k}^{n}+2 \lambda^{x} \hat{f}\left(U_{J, k}^{n}, U_{J-1, k}^{n}\right)-2 \mu^{x} \Delta_{-}^{x} A_{J, k}^{n}, \quad 1 \leq k \leq K .
\end{array}\right. \\
& \left\{\begin{array}{l}
W_{j, k}^{n+1}=U_{j, k}^{n}-2 \lambda^{y} \Delta_{-}^{y} \hat{g}\left(U_{j, k+1}^{n}, U_{j, k}^{n}\right)+2 \mu^{y} \Delta_{+}^{y} \Delta_{-}^{y} A_{j, k}^{n}, \quad 2 \leq k \leq K-1,1 \leq j \leq J, \\
W_{j, 1}^{n+1}=U_{j, k}^{n}-2 \lambda^{y} \hat{g}\left(U_{j, 2}^{n}, U_{j, 1}^{n}\right)+2 \mu^{y} \Delta_{+}^{y} A_{j, 1}^{n}, \quad 1 \leq j \leq J, \\
W_{j, K}^{n+1}=U_{j, k}^{n}+2 \lambda^{y} \hat{g}\left(U_{j, K}^{n}, U_{j, K-1}^{n}\right)-2 \mu^{y} \Delta_{-}^{y} A_{j, K}^{n}, \quad 1 \leq j \leq J .
\end{array}\right.
\end{aligned}
$$




$$
U_{j, k}^{n+1}=(1 / 2) V_{j, k}^{n+1}+(1 / 2) W_{j, k}^{n+1} .
$$

Here we have used the notation $A_{j, k}^{n}=A\left(U_{j, k}^{n}\right)$. The CFL condition 4.1 must be modified. It becomes

For all $u, v, z, w \in[0,1]$,

$$
\begin{aligned}
& -\lambda^{x} \frac{\hat{f}(v, w)-\hat{f}(u, w)}{v-u}+\lambda^{x} \frac{\hat{f}(z, v)-\hat{f}(z, u)}{v-u}+2 \mu^{x} \frac{A(v)-A(u)}{v-u} \leq \frac{1}{2}, \\
& -\lambda^{y} \frac{\hat{g}(v, w)-\hat{g}(u, w)}{v-u}+\lambda^{y} \frac{\hat{g}(z, v)-\hat{g}(z, u)}{v-u}+2 \mu^{y} \frac{A(v)-A(u)}{v-u} \leq \frac{1}{2} .
\end{aligned}
$$

Remark 6.1. The scheme 6.2), (6.3), 6.4 is essentially the explicit version of the implicit finite volume scheme of references [2] and 14, in the special case where the control volumes are rectangles arranged as a Cartesian mesh. We use the word "essentially" because of our flux modification (3.4), which does not appear in 2, 14].

Proposition 6.1. Lemmas 4.1 and 4.3 remain valid for the approximate solutions $U_{j, k}^{n}$ produced by the modified scheme 6.2, 66.3), 6.4).

Proof. The proof of Lemma 4.1 requires only straightforward modifications. We simply mention that the key new ingredients are the fact that $A(\cdot)$ is nondecreasing, and the CFL condition (6.5).

The proof of Lemma 4.3 is unchanged, except that one replaces the incremental coefficients $C_{j+\frac{1}{2}}^{n}, D_{j+\frac{1}{2}}^{n}$ by

$$
\tilde{C}_{j+\frac{1}{2}}^{n}=C_{j+\frac{1}{2}}^{n}+\mu^{x} \frac{\Delta_{+}^{x} A_{j}^{n}}{\Delta_{+}^{x} U_{j}^{n}}, \quad \tilde{D}_{j+\frac{1}{2}}^{n}=D_{j+\frac{1}{2}}^{n}+\mu^{x} \frac{\Delta_{+}^{x} A_{j}^{n}}{\Delta_{+}^{x} U_{j}^{n}} .
$$

We must check that

$$
\tilde{C}_{j+\frac{1}{2}}^{n} \geq 0, \quad \tilde{D}_{j+\frac{1}{2}}^{n} \geq 0, \quad \tilde{C}_{j+\frac{1}{2}}^{n}+\tilde{D}_{j+\frac{1}{2}}^{n} \leq 1
$$

but these inequalities follow from the assumption that $A$ is nondecreasing, along with the CFL condition 6.5.

Proposition 6.2. Recall the discrete entropy fluxes $\hat{F}$ and $\hat{G}$ defined by 4.21. For every $\kappa \in \mathbb{R}$, we have the following discrete entropy inequality:

$$
\left|U_{j, k}^{n+1}-\kappa\right| \leq\left|U_{j, k}^{n}-\kappa\right|-\Delta_{-}^{x} R_{j+\frac{1}{2}, k}^{n}-\Delta_{-}^{y} S_{j, k+\frac{1}{2}}^{n},
$$

where

$$
\begin{gathered}
R_{j+\frac{1}{2}, k}^{n}= \begin{cases}\lambda^{x} \hat{F}\left(U_{j+1, k}^{n}, U_{j, k}^{n}\right)-\mu^{x} \Delta_{+}^{x}\left|A_{j, k}^{n}-A(\kappa)\right|, & 1<j<J, \\
\lambda^{x} f(\kappa), & j=0, \\
-\lambda^{x} f(\kappa), & j=J,\end{cases} \\
S_{j, k+\frac{1}{2}}^{n}= \begin{cases}\lambda^{y} \hat{G}\left(U_{j, k+1}^{n}, U_{j, k}^{n}\right)-\mu^{y} \Delta_{+}^{y}\left|A_{j, k}^{n}-A(\kappa)\right|, & 1<k<K, \\
\lambda^{y} g(\kappa), & k=0, \\
-\lambda^{y} g(\kappa), & k=K .\end{cases}
\end{gathered}
$$

Proof. For a grid point $\left(x_{j}, y_{k}\right) \in \Omega$ that is away from $\partial \Omega$, i.e., $\left(x_{j \pm 1}, y_{k}\right),\left(x_{j}, y_{k \pm 1}\right) \in \Omega$, the proof is a straightforward extension to two dimensions of Lemma 3.7 of [13], the main ingredient being monotonicity.

Now consider a boundary grid point. For example, take the case of $\left(x_{1}, y_{k}\right) \in \Omega$, where $2 \leq$ $k \leq K-1$. Proceeding as in [13, one finds that

$$
\left|U_{1, k}^{n+1}-\kappa+\lambda^{x} f(\kappa)\right| \leq\left|U_{1, k}^{n}-\kappa\right|-R_{\frac{3}{2}, k}^{n}-\Delta_{-}^{y} S_{1, k+\frac{1}{2}}^{n} .
$$


Recalling that $f(\kappa) \geq 0$, it is easy to check that

$$
\begin{aligned}
\left|U_{1, k}^{n+1}-\kappa+\lambda^{x} f(\kappa)\right| & \geq\left|U_{1, k}^{n+1}-\kappa\right|+\lambda^{x} \operatorname{sign}\left(U_{1, k}^{n+1}-\kappa\right) f(\kappa) \\
& \geq\left|U_{1, k}^{n+1}-\kappa\right|-\lambda^{x} f(\kappa) \\
& =\left|U_{1, k}^{n+1}-\kappa\right|-R_{\frac{1}{2}, k}^{n} .
\end{aligned}
$$

Inserting this last inequality into $(6.9)$, we get the discrete entropy inequality 6.6 .

The remaining boundary cases, i.e., $j=J, k=1$, and $k=K$ are handled in a similar manner. We omit the details.

Remark 6.2. From the proof above, it is evident that we could have alternatively defined the boundary contributions as

$$
\begin{aligned}
& R_{\frac{1}{2}, k}^{n}=-\lambda^{x} \operatorname{sign}\left(U_{1, k}^{n+1}-\kappa\right) f(\kappa), \quad R_{J+\frac{1}{2}, k}^{n}=\lambda^{x} \operatorname{sign}\left(U_{J, k}^{n+1}-\kappa\right) f(\kappa), \\
& S_{j, \frac{1}{2}}^{n}=-\lambda^{y} \operatorname{sign}\left(U_{j, 1}^{n+1}-\kappa\right) g(\kappa), \quad S_{j, K+\frac{1}{2}}^{n}=\lambda^{y} \operatorname{sign}\left(U_{j, K}^{n+1}-\kappa\right) g(\kappa) .
\end{aligned}
$$

The boundary terms defined by (6.7) and (6.8) are consistent with the discrete entropy inequality of [2], while those of (6.10) are consistent with the discrete entropy inequality of [14]. Referring back to the purely hyperbolic problem, if we ignore the diffusion terms, then the boundary contributions defined by 6.10 give a discrete entropy inequality similar to the one in Definition 2.2 For our purposes the (6.7), 6.8 version is preferable because it is difficult to prove that 6.10 converges to its continuous counterpart when the mesh size approaches zero.

At this point, we specialize to the case of one space dimension, so that the spatial domain is an interval $\Omega=\left(0, a_{1}\right)$. The difference scheme simplifies to

$$
\left\{\begin{array}{l}
U_{j}^{n+1}=U_{j}^{n}-\lambda^{x} \Delta_{-}^{x} \hat{f}\left(U_{j+1}^{n}, U_{j}^{n}\right)+\mu^{x} \Delta_{+}^{x} \Delta_{-}^{x} A_{j}^{n}, \quad 2 \leq j \leq J-1, \\
U_{1}^{n+1}=U_{1}^{n}-\lambda^{x} \hat{f}\left(U_{2}^{n}, U_{1}^{n}\right)+\mu^{x} \Delta_{+}^{x} A_{1}^{n} \\
U_{J}^{n+1}=U_{J}^{n}+\lambda^{x} \hat{f}\left(U_{J}^{n}, U_{J-1}^{n}\right)-\mu^{x} \Delta_{-}^{x} A_{J}^{n}
\end{array}\right.
$$

with the data initialized via

$$
U_{j}^{0}=\frac{1}{\Delta x} \int_{I_{j}^{x}} u_{0}(x) d x,
$$

and the approximate solution extended to all of $Q_{T}$ using

$$
u^{\Delta}(x, t)=\sum_{n=0}^{N} \sum_{j=1}^{J} \chi_{j}(x) \chi^{n}(t) U_{j}^{n}, \quad(x, t) \in Q_{T},
$$

where $\Delta=(\Delta x, \Delta t)$. We will assume the following CFL condition:

For all $u, v, z, w \in[0,1]$,

$$
-\lambda^{x} \frac{\hat{f}(v, w)-\hat{f}(u, w)}{v-u}+\lambda^{x} \frac{\hat{f}(z, v)-\hat{f}(z, u)}{v-u}+2 \mu^{x} \frac{A(v)-A(u)}{v-u} \leq 1 / 2 .
$$

For the Cauchy problem associated with the one-dimensional version of the PDE of (6.1), convergence of monotone schemes was proven in [13. For grid points away from the boundary, our difference scheme is essentially the same as that of [13. Following that reference we assume, in addition to our previous assumptions, that

$$
\left|f\left(u_{0}\right)-A\left(u_{0}\right)_{x}\right| \in \mathrm{BV} .
$$

We will use the following definition of entropy solution, due to Andreianov and Gazibo [1, Definition 2.3]. We have specialized it to the one-dimensional setting, and used the fact that $|f(\kappa) \cdot \nu|=f(\kappa)$, since $f(\cdot) \geq 0$. 
Definition 6.1. A function $u \in L^{\infty}\left(Q_{T}\right)$ taking values in $[0,1]$ is called an entropy solution of (the one-dimensional version of) (6.1) if $A(u) \in L^{2}\left(0, T ; H^{1}(\Omega)\right.$ ) and if the following entropy inequality holds $\forall \kappa \in \mathbb{R}, \forall \phi \in C_{c}^{\infty}\left(\underline{\Pi}_{T}\right), \phi \geq 0$ :

$$
\begin{gathered}
\int_{0}^{T} \int_{0}^{a_{1}}\left\{|u-\kappa| \phi_{t}+\operatorname{sign}(u-\kappa)\left(f(u)-f(\kappa)-A(u)_{x}\right) \phi_{x}\right\} d x d t \\
\quad+\int_{0}^{a_{1}}\left|u_{0}-\kappa\right| \phi(x, 0) d x+\int_{0}^{T} f(\kappa)\left(\phi(0, t)+\phi\left(a_{1}, t\right)\right) d t \geq 0 .
\end{gathered}
$$

We will rely on the uniqueness portion of the following result, due to Andreianov and Gazibo.

Theorem 6.1 ([1, Theorem 4.1.]). The one-dimensional version of the problem (6.1) admits a unique entropy solution in the sense of Definition 6.1.

For a fixed mesh size $\Delta$, let

$$
\hat{z}(v, u)=\hat{f}(v, u)-(A(v)-A(u)) / \Delta x,
$$

and note that $\hat{z}$ is nonincreasing as a function of $v$, nondecreasing as a function of $u$. We will use the notation $\hat{z}_{v}\left(\hat{z}_{u}\right)$ for the partial derivative of $\hat{z}$ with respect to its first (second) argument. Define

$$
Z_{j+\frac{1}{2}}^{n}=\left\{\begin{array}{ll}
\hat{z}\left(U_{j+1}^{n}, U_{j}^{n}\right), & j=1, \ldots, J-1, \\
0, & j=0, J,
\end{array}, \quad D A_{j+\frac{1}{2}}^{n}= \begin{cases}\Delta_{+}^{x} A_{j}^{n} / \Delta x, & j=1, \ldots, J-1, \\
0, & j=0, J .\end{cases}\right.
$$

We measure the total variation of these quantities using:

$$
\operatorname{TV}\left(Z^{n}\right)=\sum_{j=0}^{J-1}\left|\Delta_{+}^{x} Z_{j+\frac{1}{2}}^{n}\right|, \quad \operatorname{TV}\left(D A^{n}\right)=\sum_{j=0}^{J-1}\left|\Delta_{+}^{x} D A_{j+\frac{1}{2}}^{n}\right|
$$

Remark 6.3. By including the terms $\left|\Delta_{+}^{x} Z_{\frac{1}{2}}^{n}\right|=\left|Z_{\frac{3}{2}}^{n}\right|$ and $\left|\Delta_{+}^{x} Z_{J-\frac{1}{2}}^{n}\right|=\left|Z_{J-\frac{1}{2}}^{n}\right|$ in the first definition above, $\operatorname{TV}\left(Z^{n}\right)$ is nonincreasing.

Lemma 6.1. We have an $L^{\infty}$ bound and a spatial $B V$ bound for $Z_{j+\frac{1}{2}}^{n}$ and thus also $D A_{j+\frac{1}{2}}^{n}$ :

$$
\max _{0 \leq j \leq J}\left|Z_{j+\frac{1}{2}}^{n}\right| \leq \max _{0 \leq j \leq J}\left|Z_{j+\frac{1}{2}}^{0}\right|, \quad T V\left(Z^{n}\right) \leq T V\left(Z^{0}\right),
$$

and

$$
\max _{0 \leq j \leq J}\left|D A_{j+\frac{1}{2}}^{n}\right| \leq C_{1}, \quad T V\left(D A^{n}\right) \leq C_{2},
$$

where the constants $C_{1}$ and $C_{2}$ are independent of the mesh size $\Delta$.

Proof. The one-dimensional scheme 6.11 can be written

$$
U_{j}^{n+1}-U_{j}^{n}=-\lambda^{x} \Delta_{-}^{x} Z_{j+\frac{1}{2}}^{n}
$$

We use 6.15 and 6.18 to derive an equation for the time evolution of $Z_{j+\frac{1}{2}}^{n}$, which is valid for $j=1, \ldots, J-1$ :

$$
\begin{aligned}
Z_{j+\frac{1}{2}}^{n+1} & =\hat{z}\left(U_{j+1}^{n+1}, U_{j}^{n+1}\right) \\
& =\hat{z}\left(U_{j+1}^{n}, U_{j}^{n}\right)+\int_{0}^{1} \frac{d}{d \theta} \hat{z}\left(U_{j+1}^{n}+\theta\left(U_{j+1}^{n+1}-U_{j+1}^{n}\right), U_{j}^{n}+\theta\left(U_{j}^{n+1}-U_{j}^{n}\right)\right) d \theta \\
& =Z_{j+\frac{1}{2}}^{n}+C_{j+\frac{1}{2}}^{n} \Delta_{+}^{x} Z_{j+\frac{1}{2}}^{n}-D_{j-\frac{1}{2}}^{n} \Delta_{+}^{x} Z_{j-\frac{1}{2}}^{n},
\end{aligned}
$$

where

$$
\begin{aligned}
& C_{j+\frac{1}{2}}^{n}=-\lambda \int_{0}^{1} \hat{z}_{v}\left(U_{j+1}^{n}+\theta\left(U_{j+1}^{n+1}-U_{j+1}^{n}\right), U_{j}^{n}+\theta\left(U_{j}^{n+1}-U_{j}^{n}\right)\right) d \theta \geq 0, \\
& D_{j-\frac{1}{2}}^{n}=\lambda \int_{0}^{1} \hat{z}_{u}\left(U_{j+1}^{n}+\theta\left(U_{j+1}^{n+1}-U_{j+1}^{n}\right), U_{j}^{n}+\theta\left(U_{j}^{n+1}-U_{j}^{n}\right)\right) d \theta \geq 0 .
\end{aligned}
$$


With the CFL condition 6.14, we have

$$
0 \leq C_{j+\frac{1}{2}}^{n} \leq \frac{1}{2}, \quad 0 \leq D_{j-\frac{1}{2}}^{n} \leq \frac{1}{2}
$$

This, along with the incremental form 6.19 implies that $Z_{j+\frac{1}{2}}^{n+1}$ is a convex combination of $Z_{j+\frac{1}{2}}^{n}$, $Z_{j-\frac{1}{2}}^{n}, Z_{\mathrm{J}+\frac{3}{2}}^{n}$, which gives the $L^{\infty}$ bound of $\left[6.16\right.$ for $1 \leq j \leq J-1$. When $j=0, J$, the $L^{\infty}$ bound is immediate, since $Z_{\frac{1}{2}}^{n}=Z_{J+\frac{1}{2}}^{n}=0$.

Next, we take spatial differences of the incremental form 6.19):

$$
\Delta_{+}^{x} Z_{j+\frac{1}{2}}^{n+1}=\left(1-C_{j+\frac{1}{2}}^{n}-D_{j+\frac{1}{2}}^{n}\right) \Delta_{+}^{x} Z_{j+\frac{1}{2}}^{n}+C_{j+\frac{3}{2}}^{n} \Delta_{+}^{x} Z_{j+\frac{3}{2}}^{n}+D_{j-\frac{1}{2}}^{n} \Delta_{+}^{x} Z_{j-\frac{1}{2}}^{n} \cdot
$$

This formula is valid for $1 \leq j \leq J-2$. For $j=0, J-1$ we use the fact that

$$
Z_{\frac{1}{2}}^{n+1}=Z_{\frac{1}{2}}^{n}=0, \quad Z_{J+\frac{1}{2}}^{n+1}=Z_{J+\frac{1}{2}}^{n}=0 .
$$

Then,

$$
\begin{aligned}
\Delta_{+}^{x} Z_{\frac{1}{2}}^{n+1} & =Z_{\frac{3}{2}}^{n+1}-Z_{\frac{1}{2}}^{n+1}=Z_{\frac{3}{2}}^{n}+C_{\frac{3}{2}}^{n} \Delta_{+}^{x} Z_{\frac{3}{2}}^{n}-D_{\frac{1}{2}}^{n} \Delta_{+}^{x} Z_{\frac{1}{2}}^{n}-Z_{\frac{1}{2}}^{n} \\
& =\left(1-D_{\frac{1}{2}}^{n}\right) \Delta_{+}^{x} Z_{\frac{1}{2}}^{n}+C_{\frac{3}{2}}^{n} \Delta_{+}^{x} Z_{\frac{3}{2}}^{n}, \\
\Delta_{+}^{x} Z_{J-\frac{1}{2}}^{n+1} & =Z_{J+\frac{1}{2}}^{n+1}-Z_{J-\frac{1}{2}}^{n+1}=Z_{J+\frac{1}{2}}^{n}-\left(Z_{J-\frac{1}{2}}^{n}+C_{J-\frac{1}{2}}^{n} \Delta_{+}^{x} Z_{J-\frac{1}{2}}^{n}-D_{J-\frac{3}{2}}^{n} \Delta_{+}^{x} Z_{J-\frac{3}{2}}^{n}\right) \\
& =\left(1-C_{J-\frac{1}{2}}^{n}\right) \Delta_{+}^{x} Z_{J-\frac{1}{2}}^{n}+D_{J-\frac{3}{2}}^{n} \Delta_{+}^{x} Z_{J-\frac{3}{2}}^{n} .
\end{aligned}
$$

Taking absolute values of 6.21 and 6.22, then using 6.20, summing over $j$, and canceling telescoping terms, we get

$$
\sum_{j=0}^{J-1}\left|\Delta_{+}^{x} Z_{j+\frac{1}{2}}^{n+1}\right| \leq \sum_{j=0}^{J-1}\left|\Delta_{+}^{x} Z_{j+\frac{1}{2}}^{n}\right| \leq \ldots \leq \sum_{j=0}^{J-1}\left|\Delta_{+}^{x} Z_{j+\frac{1}{2}}^{0}\right|,
$$

which gives the spatial variation bound of 6.16 .

For the bounds 6.17), note that $D A_{j+\frac{1}{2}}^{n}=f\left(U_{j+1}^{n}, U_{j}^{n}\right)-Z_{j+\frac{1}{2}}^{n}$. Both the $L^{\infty}$ bound and the total variation bound then follow from the Lipschitz continuity of $\hat{f}$, along with the one-dimensional version of Proposition 6.1 and the bounds 6.16.

We have the following convergence theorem for our one-dimensional scheme.

Theorem 6.2. The approximate solutions $\left\{u^{\Delta}\right\}_{\Delta>0}$ defined by the one-dimensional scheme (6.11), 6.12 , 6.13 converge in $L_{\mathrm{loc}}^{1}\left(Q_{T}\right)$ and boundedly a.e. in $Q_{T}$ to

$$
u \in L^{\infty}\left(Q_{T}\right) \cap B V\left(Q_{T}\right) .
$$

In addition,

$$
A(u)_{x} \in L^{\infty}\left(Q_{T}\right) \text { and thus } A(u) \in L^{2}\left(0, T ; H^{1}(\Omega)\right) .
$$

The limit function $u$ is the unique entropy solution to the initial boundary problem (6.1).

Proof. Using the BV bound of Lemma 6.1, we get the following discrete time continuity bound by repeating Lemma 3.5 of [13]:

$$
\sum_{j=1}^{J}\left|U_{j}^{m}-U_{j}^{n}\right| \leq \operatorname{TV}\left(Z^{0}\right) \frac{\Delta t}{\Delta x}|m-n| .
$$

Using the bounds provided by the one-dimensional version of Proposition 6.1, along with (6.24, one gets convergence along a subsequence in $L_{\text {loc }}^{1}\left(Q_{T}\right)$ and boundedly a.e. in $Q_{T}$, to some function $u \in L^{\infty}\left(Q_{T}\right) \cap \operatorname{BV}\left(Q_{T}\right)$. Since $z \mapsto A(z)$ is Lipschitz-continuous, for the same subsequence we also have $A\left(u^{\Delta}\right) \rightarrow A(u) \in L^{\infty}\left(Q_{T}\right) \cap \mathrm{BV}\left(Q_{T}\right)$. For this same subsequential limit $u$, the assertion 6.23 then follows from 6.17 of Lemma 6.1.

Next, we repeat the Lax-Wendroff type calculation found on page 1855 of [13], starting from the one-dimensional version of the discrete entropy inequality (6.6), and taking into account the boundary contributions at $j=1$ and $j=J$. We conclude that the subsequential limit $u$ satisfies 
the entropy inequality 6.1, and therefore by Theorem 6.1 $u$ is the unique entropy solution of 6.1). Finally, by uniqueness we have convergence to $u$ of the entire computed sequence $u^{\Delta}$, not just a subsequence.

Remark 6.4. Although strong traces of the total flux are not required for Definition 6.1, we can prove their existence for this one-dimensional problem. In fact, by applying the test function argument in the proof of Lemma 3.6 of [13, we get

$$
\Delta x \sum_{j=0}^{J}\left|Z_{j+\frac{1}{2}}^{m}-Z_{j+\frac{1}{2}}^{n}\right| \leq C \sqrt{(m-n) \Delta t} .
$$

Using this $L^{1}$ time continuity result, along with Lemma 6.1. we have convergence of $\left\{z^{\Delta}\right\}$ to $z=f(u)-A(u)_{x} \in L^{\infty}\left(Q_{T}\right) \cap L^{\infty}(0, T ; \mathrm{BV}(\Omega))$. Thus the total flux satisfies

$$
f(u)-A(u)_{x} \in L^{\infty}\left(Q_{T}\right) \cap L^{\infty}(0, T ; \mathrm{BV}(\Omega)) .
$$

This implies that the boundary trace of $f(u)-A(u)_{x}$ exists. In addition, thanks to 6.25),

$$
\left\|\left(f(u)-A(u)_{x}\right)\left(\cdot, t_{2}\right)-\left(f(u)-A(u)_{x}\right)\left(\cdot, t_{1}\right)\right\|_{L^{1}(\Omega)} \leq C \sqrt{t_{2}-t_{1}} .
$$

\section{ACKNOWLEDGMENTS}

This work was supported by the Research Council of Norway via grant no. 250674/F20. We would like to thank the anonymous referees for valuable comments.

\section{REFERENCES}

[1] B. Andreianov and M. K. Gazibo. Entropy formulation of degenerate parabolic equation with zero-flux boundary condition. Z. Angew. Math. Phys., 64(5):1471-1491, 2013.

[2] B. Andreianov and M. K. Gazibo. Convergence of finite volume scheme for degenerate parabolic problem with zero flux boundary condition. In Finite volumes for complex applications. VII. Methods and theoretical aspects, volume 77 of Springer Proc. Math. Stat., pages 303-311. Springer, Cham, 2014.

[3] B. Andreianov and K. Sbihi. Well-posedness of general boundary-value problems for scalar conservation laws. Trans. Amer. Math. Soc., 367(6):3763-3806, 2015.

[4] C. Bardos, A. Y. le Roux, and J.-C. Nédélec. First order quasilinear equations with boundary conditions. Comm. Partial Differential Equations, 4(9):1017-1034, 1979.

[5] R. Bürger, S. Evje, and K. H. Karlsen. On strongly degenerate convection-diffusion problems modeling sedimentation-consolidation processes. J. Math. Anal. Appl., 247(2):517-556, 2000.

[6] R. Bürger, H. Frid, and K. H. Karlsen. On a free boundary problem for a strongly degenerate quasi-linear parabolic equation with an application to a model of pressure filtration. SIAM J. Math. Anal., 34(3):611-635 (electronic), 2003.

[7] R. Bürger, H. Frid, and K. H. Karlsen. On the well-posedness of entropy solutions to conservation laws with a zero-flux boundary condition. J. Math. Anal. Appl., 326(1):108-120, 2007.

[8] R. Bürger and K. H. Karlsen. On some upwind difference schemes for the phenomenological sedimentationconsolidation model. J. Engrg. Math., 41(2-3):145-166, 2001.

[9] R. Bürger, A. García, K. H. Karlsen, and J. D. Towers. A family of numerical schemes for kinematic flows with discontinuous flux. J. Engrg. Math., 60:387-425, 2008.

[10] R. Bürger and M. Kunik. A critical look at the kinematic-wave theory for sedimentation-consolidation processes in closed vessels. Math. Models Methods Appl. Sci., 24:1257-1273, 2001.

[11] M. G. Crandall and A. Majda. Monotone difference approximations for scalar conservation laws. Math. Comp., 34(149):1-21, 1980

[12] L. C. Evans and R. F. Gariepy. Measure theory and fine properties of functions. Studies in Advanced Mathematics. CRC Press, Boca Raton, FL, 1992.

[13] S. Evje and K. H. Karlsen. Monotone difference approximations of $B V$ solutions to degenerate convectiondiffusion equations. SIAM J. Numer. Anal., 37(6):1838-1860 (electronic), 2000.

[14] M. K. Gazibo. Degenerate parabolic equation with zero flux boundary condition and its approximations. Preprint available at http://hal.archives-ouvertes.fr/hal-00855746.

[15] A. Harten, J. Hyman, and P. Lax. On finite-difference approximations and entropy conditions for shocks. Comm. Pure Appl. Math., XXIX:297-322, 1976.

[16] M. Hilliges and W. Weidlich. A phenomenological model for dynamic traffic flow in networks. Transp. Res. B, 29: 407-431, 1995.

[17] H. Holden and N. H. Risebro. Front tracking for hyperbolic conservation laws, volume 152 of Applied Mathematical Sciences. Springer-Verlag, New York, 2002. 
[18] K. H. Karlsen, K.-A. Lie, and N. H. Risebro. A front tracking method for conservation laws with boundary conditions. In Hyperbolic problems: theory, numerics, applications, Vol. I (Zürich, 1998), pages 493-502. Birkhäuser, Basel, 1999.

[19] S. N. Kružkov. First order quasi-linear equations in several independent variables. Math. USSR Sbornik, 10:217243, 1970.

[20] P.-L. Lions, B. Perthame, and E. Tadmor. A kinetic formulation of multidimensional scalar conservation laws and related equations. J. Amer. Math. Soc., 7(1):169-191, 1994.

[21] R. Sanders. On convergence of monotone finite difference schemes with variable spatial differencing. Math. Comp., 40(161):91-106, 1983. 